\title{
Gyrotropic effects in trigonal tellurium studied from first principles
}

\author{
Stepan S. Tsirkin, ${ }^{1,2}$ Pablo Aguado Puente, ${ }^{1,2}$ and Ivo Souza ${ }^{1,3}$ \\ ${ }^{1}$ Centro de Física de Materiales, Universidad del País Vasco, E-20018 San Sebastián, Spain \\ ${ }^{2}$ Donostia International Physics Centre, E-20018 San Sebastián, Spain \\ ${ }^{3}$ Ikerbasque Foundation, E-48013 Bilbao, Spain
}

(Received 10 October 2017; revised manuscript received 4 January 2018; published 29 January 2018)

\begin{abstract}
We present a combined $a b$ initio study of several gyrotropic effects in $p$-doped trigonal tellurium (effects that reverse direction with the handedness of the spiral chains in the atomic structure). The key ingredients in our study are the $k$-space Berry curvature and intrinsic orbital magnetic moment imparted on the Bloch states by the chirality of the crystal structure. We show that the observed sign reversal with temperature of the circular photogalvanic effect can be explained by the presence of Weyl points near the bottom of the conduction band acting as sources and sinks of Berry curvature. The passage of a current along the trigonal axis induces a rather small parallel magnetization, which can nevertheless be detected by optical means (Faraday rotation of transmitted light) due to the high transparency of the sample. In agreement with experiment, we find that when infrared light propagates antiparallel to the current at low doping the current-induced optical rotation enhances the natural optical rotation. According to our calculations the plane of polarization rotates in the opposite sense to the bonded atoms in the spiral chains, in agreement with a recent experiment that contradicts earlier reports.
\end{abstract}

DOI: 10.1103/PhysRevB.97.035158

\section{INTRODUCTION}

The spontaneous magnetization of ferromagnetic metals gives rise to Hall and Faraday effects at $\boldsymbol{B}=0$. These effects are termed anomalous, in opposition to the ordinary (linear in $\boldsymbol{B})$ Hall and Faraday effects in metals lacking magnetic order. The scattering-free or intrinsic contribution to the anomalous Hall conductivity (AHC) is given by $[1,2]$

$$
\begin{aligned}
\sigma_{a b}^{\mathrm{A}} & =-\frac{e^{2}}{\hbar} \int[d \boldsymbol{k}] \sum_{n} f_{0}\left(E_{\boldsymbol{k} n}, \mu, T\right) \epsilon_{a b c} \Omega_{\boldsymbol{k} n}^{c}, \\
\boldsymbol{\Omega}_{\boldsymbol{k} n} & =\nabla_{\boldsymbol{k}} \times \boldsymbol{A}_{\boldsymbol{k} n}=-\operatorname{Im}\left\langle\boldsymbol{\partial}_{\boldsymbol{k}} u_{\boldsymbol{k} n}|\times| \boldsymbol{\partial}_{\boldsymbol{k}} u_{\boldsymbol{k} n}\right\rangle,
\end{aligned}
$$

where $\boldsymbol{A}_{\boldsymbol{k} n}=i\left\langle u_{\boldsymbol{k} n} \mid \partial_{\boldsymbol{k}} u_{\boldsymbol{k} n}\right\rangle$ is the Berry connection, $\boldsymbol{\Omega}_{\boldsymbol{k} n}$ is the Berry curvature, $E_{k n}$ is the band energy, $f_{0}$ is the equilibrium occupation factor, and the integral is over the Brillouin zone with $[d \boldsymbol{k}] \equiv d^{3} k /(2 \pi)^{3}$.

The possibility of inducing similar effects in nonmagnetic conductors by purely electrical means was raised by Baranova et al. [3], who predicted the existence of an electrical analog of the Faraday effect in chiral conducting liquids: a change in rotatory power caused by the passage of an electrical current. In the following, we shall refer to this phenomenom as "kinetic Faraday effect" (kFE). ${ }^{1}$ In the kFE the induced rotatory power reverses sign with the applied electric field $\mathcal{E}$, in much the same way that in the ordinary Faraday effect it reverses sign with $\boldsymbol{B}$. Although it has not been observed so far in liquids, the $\mathrm{kFE}$

\footnotetext{
${ }^{1}$ Although this is a nonstandard designation, we find it preferable to current-induced optical activity [4,5] since the effect is closer to Faraday rotation than to natural optical activity. The name adopted here is also consistent with that of a closely related phenomenom to be discussed shortly, the kinetic magnetoelectric effect.
}

was measured in a chiral conducting crystal, $p$-doped trigonal Te [4,5], following a theoretical prediction [6]. The effect is symmetry allowed in the 18 (out of 21) acentric crystal classes known as gyrotropic [7], including those for which natural optical rotation is disallowed.

Gyrotropic crystals also display a nonlinear optical effect closely related to the $\mathrm{kFE}$ : the circular photogalvanic effect (CPGE). It consists in the generation of a photocurrent that reverses sign with the helicity of light [6-11], and occurs when light is absorbed via interband or intraband scattering processes, with the latter involving virtual transitions to other bands [11].

When impurity scattering is treated in the constant relaxation-time approximation, it becomes possible to identify a contribution to the intraband CPGE associated with the Berry curvature of the free carriers [12-14]. This "intrinsic" contribution, proportional to the relaxation time $\tau$, is conveniently described in terms of the following dimensionless tensor,

$$
D_{a b}=\int[d \boldsymbol{k}] \sum_{n} \frac{\partial E_{\boldsymbol{k} n}}{\partial k_{a}} \Omega_{\boldsymbol{k} n}^{b}\left(-\frac{\partial f_{0}}{\partial E}\right)_{E=E_{\boldsymbol{k} n}},
$$

where the index $\boldsymbol{k}$ has been dropped for brevity. $D$ transforms like the gyration tensor $g$, but unlike $g$ it is always traceless. ${ }^{2}$ This means that $D$ can only be nonzero in 16 of the 18 gyrotropic crystal classes; the excluded classes are $\mathrm{O}$ and $\mathrm{T}$, for which $g$ is isotropic (its form is tabulated in Ref. [10] for all the gyrotropic crystal classes).

\footnotetext{
${ }^{2}$ After integrating Eq. (2) by parts, the trace of the tensor $D$ can be expressed as a Brillouin-zone integral of the divergence of the Berry curvature, weighted by the occupation factor. The fact that the Berry curvature is divergence-free except at isolated chiral band crossings (Weyl points) implies that $D$ is traceless [12].
} 

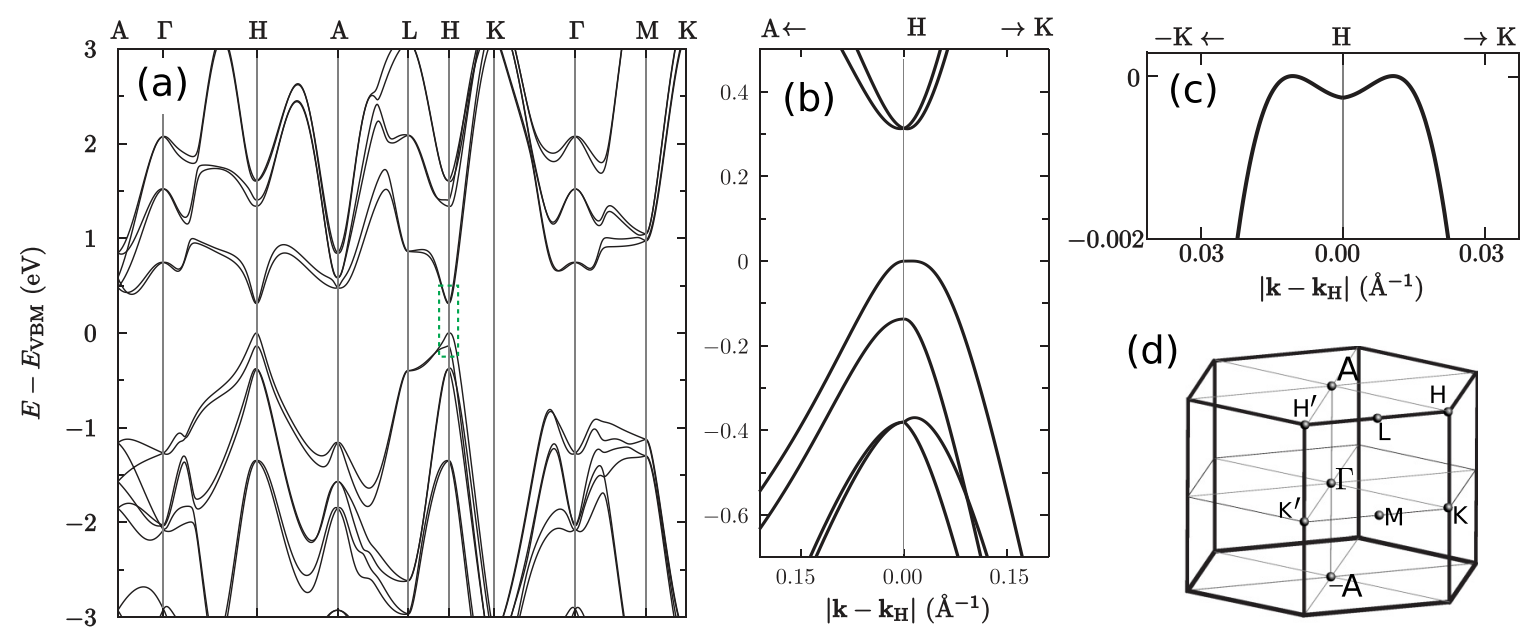

(d)

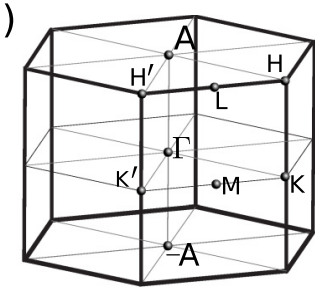

FIG. 1. Fully relativistic band structure of trigonal Te, with energies measured from the valence-band maximum (VBM). (b) A blow-up of the region demarcated by a dashed rectangle in (a), and (c) shows the top of the upper valence band around $\mathrm{H}$, along the HK line. The Brillouin zone and its high-symmetry points are displayed in (d).

In addition to the CPGE, the tensor $D$ also describes a nonlinear anomalous Hall effect (AHE) [14] that can be viewed as the low-frequency limit of the kFE. Indeed, the kFE is governed by a tensor $\widetilde{D}(\omega)$ [Eq. (12) below] that reduces to $D$ at $\omega=0$.

The flow of electrical current that gives rise to the $\mathrm{kFE}$ generates a net magnetization in the gyrotropic medium, a phenomenom known as kinetic magnetoelectric effect (kME) [15]. It was first proposed for bulk chiral conductors $[6,15]$ and later for two-dimensional (2D) inversion layers [16,17], where it has been studied intensively [18].

A microscopic theory of the intrinsic kME effect in bulk crystals was recently developed $[19,20]$. The response, proportional to $\tau$, is described by

$$
K_{a b}=\int[d \boldsymbol{k}] \sum_{n} \frac{\partial E_{k n}}{\partial k_{a}} m_{\boldsymbol{k} n}^{b}\left(-\frac{\partial f_{0}}{\partial E}\right)_{E=E_{k n}},
$$

which has the same form as Eq. (2) but with the Berry curvature replaced by the intrinsic magnetic moment $\boldsymbol{m}_{\boldsymbol{k} n}$ of the Bloch electrons. In addition to the spin moment, $\boldsymbol{m}_{\boldsymbol{k} n}$ has an orbital component given by [2]

$$
\boldsymbol{m}_{k n}^{\text {orb }}=\frac{e}{2 \hbar} \operatorname{Im}\left\langle\partial_{k} u_{k n}\left|\times\left(H_{k}-E_{k n}\right)\right| \partial_{k} u_{k n}\right\rangle,
$$

where we chose $e>0$. The tensor $K$ (with units of amperes) is symmetry allowed in all 18 gyrotropic crystal classes, and its symmetric part gives an intraband contribution to natural optical rotation at low frequencies [20,21].

In this work, we evaluate from first principles in $p$-doped tellurium ( $p$-Te) the CPGE and nonlinear AHE described by the tensor $D$, the kFE described by $\widetilde{D}(\omega)$, and the kME and intraband natural optical activity described by $K$, as well as the interband natural optical activity. We study them as a function of temperature and acceptor concentration, compare with the available experimental data, and establish correlations between them on the basis of a unified microscopic picture.

The manuscript is organized as follows. In Sec. II we describe the crystal structure of trigonal Te, the energy bands, and the form of the gyrotropic response tensors. In the subsequent sections we present and analyze our first-principles results for the various gyrotropic effects. The circular photogalvanic effect is treated in Sec. III, the nonlinear anomalous Hall effect in Sec. IV, the kinetic Faraday effect in Sec. V, the kinetic magnetoelectric effect in Sec. VI, and natural optical activity in Sec. VII. In each section, only the essential theory needed to understand the results under discussion is given; all derivations and additional technical details are left to the Appendixes.

\section{CRYSTAL STRUCTURE, ENERGY BANDS, AND SYMMETRY CONSIDERATIONS}

Elemental Te is a nonmagnetic semiconductor that crystalizes in two enantiomorphic structures with space groups $\mathrm{P} 3_{1} 21$ and $\mathrm{P}_{2} 21$ (crystal class 32 ). The unit cell contains three atoms disposed along a spiral chain that is right-handed for $\mathrm{P} 3_{1} 21$ and left-handed for $\mathrm{P}_{2} 21$, with the chains arranged on a hexagonal net. In addition to the screw symmetry along the trigonal axis, there are three twofold axes lying on the perpendicular plane.

The calculations reported in this work were carried out for the right-handed Te structure described in Ref. [22]. For the left-handed enantiomorph, the tensors $D$ and $K$ flip sign. These two tensors assume the forms,

$$
D=\frac{D_{\|}}{2}\left(\begin{array}{ccc}
-1 & 0 & 0 \\
0 & -1 & 0 \\
0 & 0 & 2
\end{array}\right)
$$

(note that the trace vanishes ${ }^{2}$ ) and

$$
K=\left(\begin{array}{ccc}
K_{\perp} & 0 & 0 \\
0 & K_{\perp} & 0 \\
0 & 0 & K_{\|}
\end{array}\right),
$$

where $\|$ and $\perp$ denote the directions parallel and perpendicular to the trigonal axis, respectively.

The fully relativistic density-functional theory calculations were done using the HSE06 hybrid functional [23]. Figure 1 
shows the calculated energy bands. The energy gap of $0.312 \mathrm{eV}$ at the $\mathrm{H}$ point is in good agreement with the value of $0.314 \mathrm{eV}$ obtained with the GW method [24], and with the experimental value of $0.323 \mathrm{eV}$ [25]. The characteristic "camel-back" shape of the upper valence band around $\mathrm{H}$ can be seen in Fig. 1(c). The band structure in Fig. 1 is in good agreement with other fully relativistic calculations [24,26]. It was calculated in the same way as in Ref. [27], and we refer the reader to that work for further details.

Below room temperature, the transport and low-frequency optical properties of weakly $p$-doped Te are governed by the upper valence band together with the lower conduction subbands. The conduction subbands have an anisotropic Rashba-type spin-orbit splitting around H, visible in Fig. 1(b); their spin textures (not shown) are consistent with those reported in Ref. [24].

The three band degeneracies visible in Fig. 1(b) are Weyl points [24]. Of particular interest to the present study is the one at $\mathrm{H}$ between the conduction subbands. It has positive chirality in the right-handed structure, which means that it acts as a source (sink) of Berry curvature in the lower (upper) subband. Time-reversal symmetry maps the Weyl point at $\mathrm{H}$ onto a Weyl point of the same chirality at $\mathrm{H}^{\prime}$. More generally, it sends $\left(E_{\boldsymbol{k} n}, \boldsymbol{\Omega}_{\boldsymbol{k} n}, \boldsymbol{m}_{\boldsymbol{k} n}\right)$ to $\left(E_{-\boldsymbol{k}, n},-\boldsymbol{\Omega}_{-\boldsymbol{k}, n},-\boldsymbol{m}_{-\boldsymbol{k}, n}\right)$, so that $\boldsymbol{k}$ and $\boldsymbol{-} \boldsymbol{k}$ contribute equally to Eqs. (2) and (3).

\section{CIRCULAR PHOTOGALVANIC EFFECT}

A detailed study of the CPGE in Te due to free-carrier absorption was reported in Ref. [9]. The measurements were

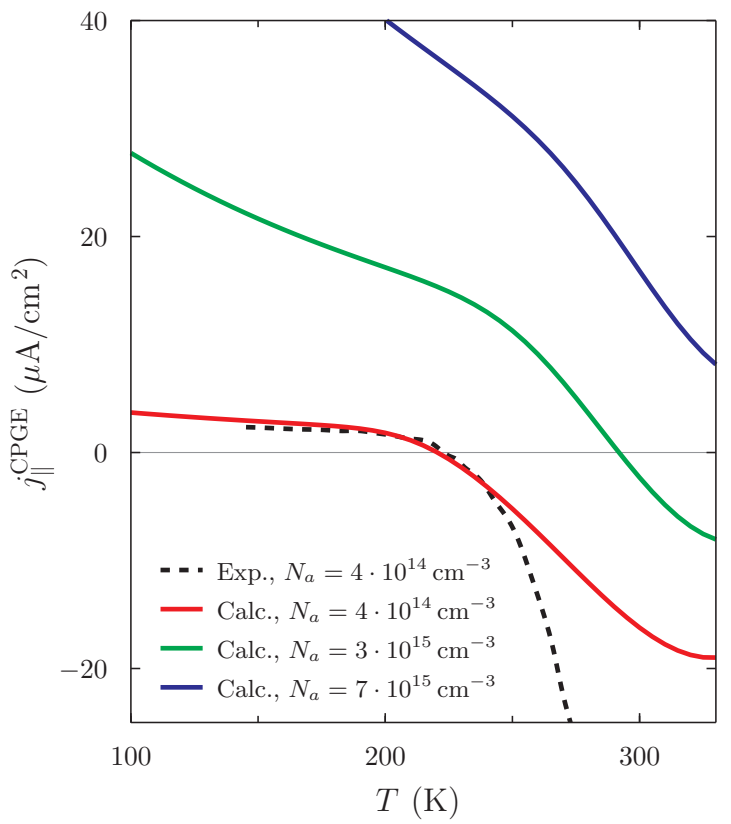

FIG. 2. (Solid lines) Temperature dependence, for different acceptor concentrations, of the intraband photocurrent density induced in right-handed Te by circularly polarized light of positive helicity and intensity $I=10 \mathrm{~W} / \mathrm{cm}^{2}$ propagating along the trigonal axis in the positive direction. According to Eq. (7), the photocurrent is proportional to $D_{\|}$. (Dashed line) Open-circuit photovoltage measured in Ref. [9], converted to a current density as described in the main text. done at room temperature and below on samples with a residual acceptor concentration $N_{a} \approx 4 \times 10^{14} \mathrm{~cm}^{-3}$, using a $\mathrm{CO}_{2}$ laser source with frequency $\hbar \omega=0.117 \mathrm{eV}$. Under these conditions the relaxation time exceeds $10^{-12} \mathrm{~s}$ [26] so that $\omega \tau \gg 1$, and Eq. (A7) for the intrinsic contribution to the intraband photocurrent becomes

$$
j_{\|}^{\mathrm{CPGE}}\left(N_{a}, T\right)=\operatorname{sgn}\left(q_{\|}\right)\left(2 \pi \mathfrak{a} \mathcal{P}_{\text {circ }} D_{\|}\right) \frac{e I_{0}}{\hbar \omega} .
$$

The quantity $D_{\|}\left(\mu\left(N_{a}, T\right), T\right)$ is given by Eqs. (2) and (5), $\mathfrak{a} \approx 1 / 137$ is the fine-structure constant, and $I_{0}$ is the intensity of incident light with wave vector $q_{\|}$and degree of circular polarization $\mathcal{P}_{\text {circ }}$ propagating along the trigonal axis.

The photocurrent density calculated from Eq. (7) with $\operatorname{sgn}\left(q_{\|}\right)>0$ and $\mathcal{P}_{\text {circ }}=+1$ is plotted versus temperature in Fig. 2 for several acceptor concentrations, assuming a laser intensity of $10 \mathrm{~W} / \mathrm{cm}^{2}$ (see below). The photocurrent starts out positive at low temperature, and becomes negative at around room temperature (except at the highest doping level). Such a sign reversal was indeed observed experimentally [9]. For a more detailed comparison, we have converted the opencircuit photovoltage and longitudinal conductivity measured in Ref. [9] into a current density, shown as a dashed curve after an overall sign change (the handedness of the sample was not determined in Ref. [9]). Since the laser intensity was also not reported, we fixed the value of $I_{0}$ in Eq. (7) by matching the experimental values at low temperature. At the experimental doping level the calculated photocurrent changes sign at around $220 \mathrm{~K}$, in good agreement with experiment.

In order to understand the temperature dependence, it is convenient to express the quantity $D_{\|}$in Eq. (7) as

$$
\begin{aligned}
D_{\|}(\mu, T) & =\int_{-\infty}^{+\infty} d \varepsilon D_{\|}^{0}(\varepsilon)\left(-\frac{\partial f_{0}(E, \mu, T)}{\partial E}\right)_{E=\varepsilon}, \\
D_{\|}^{0}(\varepsilon) & =\frac{1}{(2 \pi)^{3}} \sum_{n} \int_{E_{k n}=\varepsilon} d S \hat{v}_{k n}^{z} \Omega_{k n}^{z},
\end{aligned}
$$

where $D_{\|}^{0}(\varepsilon) \equiv D_{\|}(\varepsilon, T \approx 0)$, and $\hat{\boldsymbol{v}}_{\boldsymbol{k} n}$ is the unit vector along the band velocity. ${ }^{3}$

Figure 3(a) shows that $D_{\|}^{0}$ has opposite signs at the two band edges, increasing slowly into the valence band and rapidly into the conduction band, where it peaks. At the experimental doping level, $-\partial f_{0} / \partial E$ at $150 \mathrm{~K}$ is non-negligible in the valence band only, resulting in a positive $D_{\|}$. At $250 \mathrm{~K}$ the chemical potential $\mu$ approaches the center of the gap, and $-\partial f_{0} / \partial E$ reaches the conduction band. $D_{\|}$now collects contributions of opposite signs from the two band edges; the largest one comes from the $D_{\|}^{0}$ peak in the conduction band, which renders $D_{\|}$negative. (When $N_{a}$ is increased to

\footnotetext{
${ }^{3}$ Equation (8) is also convenient for numerical work. Once $D_{\|}^{0}(\varepsilon)$ has been calculated from Eq. (8b), Eq. (8a) can be used to evaluate $D_{\|}$as a function of $T$ and $N_{a}$ at a low computational cost. The temperature dependence of the chemical potential is calculated assuming that at the temperatures of interest all dopant levels are activated. The same approach will be used in subsequent sections to evaluate the tensors $\tilde{D}(\omega), K$, and $C$ [Eq. (B6)].
} 

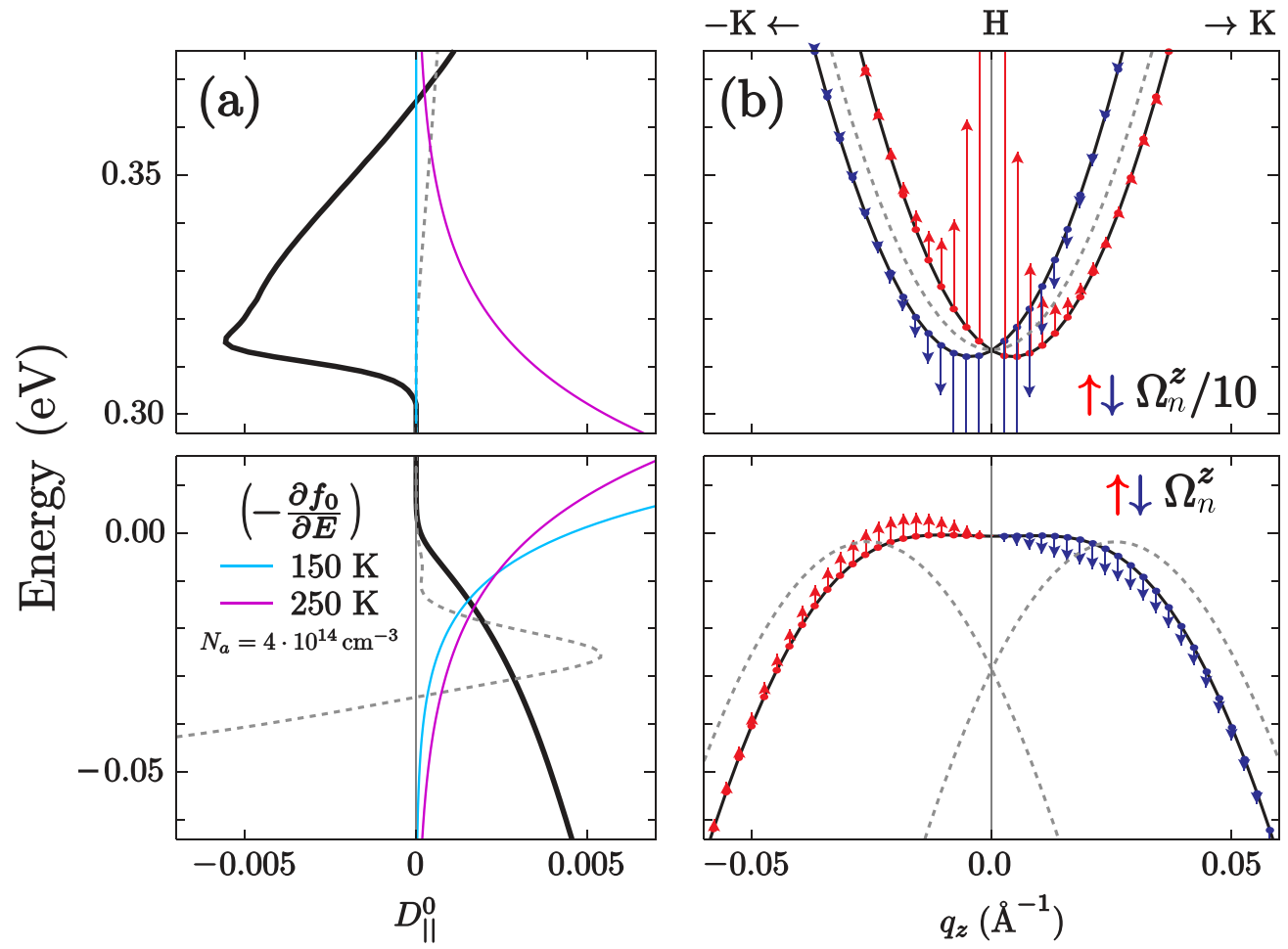

FIG. 3. Microscopic mechanism of the intraband circular photogalvanic effect in right-handed Te. (a) The quantity $D_{\|}^{0}$ in Eq. (8b) versus $\varepsilon$ measured from the VBM, calculated using a Fermi smearing of $23 \mathrm{~K}$ with (heavy black solid line) and without (dashed gray line) spin-orbit coupling. The light colored lines show the function $-\partial f_{0}\left(E, \mu\left(N_{a}, T\right), T\right) /\left.\partial E\right|_{E=\varepsilon}$ plotted versus $\varepsilon$ at fixed $N_{a}$ and two different temperatures, as detailed in the inset. (b) (Solid lines) Fully relativistic band structure in the vicinity of the $\mathrm{H}$ point. $q_{z}$ denotes $k_{z}$ measured from the $\mathrm{H}$ point along HK, and the arrows denote the $z$ component of the Berry curvature on each band. (Dashed lines) Scalar-relativistic band structure; for comparison purposes, the band edges have been aligned with those of the fully relativistic calculation.

$7 \times 10^{15} \mathrm{~cm}^{-3}, \mu$ stays close to the valence-band edge even at room temperature. The photocurrent is then dominated by holelike carriers, and it remains positive over the entire temperature range of Fig. 2.)

The behavior of $D_{\|}^{0}(\varepsilon)$ at the two band edges can be understood by inspecting the energy bands and their Berry curvatures along the HK line [Fig. 3(b)]. Because of twofold symmetry about $\Gamma \mathrm{K}, v_{k n}^{z}$ and $\Omega_{k n}^{z}$ are both odd in $q_{z}=k_{z}-k_{H}, z$, so that $q_{z}$ and $-q_{z}$ contribute equally to Eq. (8b). Regarding the $\Omega_{k n}^{z}$ profiles, note that the Berry curvature of a band arises from its coupling to other bands [see Eq. (C20)], and that this coupling becomes resonantly enhanced at (near) degeneracies $[1,2]$. At the nondegenerate valence-band edge this coupling has no singularities and as a result $\Omega_{k n}^{z}$ varies smoothly with $q_{z}$, vanishing at $q_{z}=0$. Apart from a small region between the "camel humps" that gives a negligible contribution, $\hat{v}_{\boldsymbol{k} n}^{z}$ and $\Omega_{k n}^{z}$ have the same sign, which explains the steady increase in $D_{\|}^{0}$ towards positive values as $\varepsilon$ enters the valence band.

At the edge of the conduction band the Berry curvature is dominated by the strong intersubband coupling near the Weyl point, which acts as a monopole of Berry curvature leading to $\Omega_{k n}^{z} \propto \pm q_{z}^{-2}$ for small $\left|q_{z}\right|$ [2]. When $\varepsilon$ is slightly above the crossing energy, the two subbands give competing contributions to Eq. (8b): $\left|\Omega_{k n}^{z}\right|$ is larger on the inner branch, but the outer branch has a larger energy isosurface. For an isotropic three-dimensional (3D) Rashba model these two contributions would cancel out, ${ }^{2}$ but the anisotropy of the Rashba splitting in $\mathrm{Te}$ is such that the inner branch dominates the integral in
Eq. (8b), producing a negative peak in $D_{\|}^{0}$ near the Weyl-point energy.

A minimal model for the conduction-band edge is [28]

$$
H^{\mathrm{R}}(\mathbf{q})=\frac{\hbar^{2} q_{\|}^{2}}{2 m_{\|}}+\frac{\hbar q_{\perp}^{2}}{2 m_{\perp}}+\hbar v_{\|} q_{\|} \sigma_{z}+\hbar v_{\perp}\left(q_{x} \sigma_{x}+q_{y} \sigma_{y}\right),
$$

where $\boldsymbol{q}=\boldsymbol{k}-\boldsymbol{k}_{\mathrm{H}}$. We have evaluated Eq. (8b) numerically for this two-band model, starting from the analytic expression for the Berry curvature [29]. As expected $D_{\|}^{0}$ vanishes in the isotropic limit, and when either $m_{\|} \neq m_{\perp}$ or $v_{\|} \neq v_{\perp}$ a peak develops around the Weyl crossing. For a given chirality, the peak can change sign depending on the ratios $m_{\|} / m_{\perp}$ and $v_{\|} / v_{\perp}$, as illustrated in Fig. 4.

While spin-orbit coupling is not needed to generate Weyl points and Berry curvatures in the bands of Te (in contrast to centrosymmetric collinear ferromagnets, where it is essential), the intraband CPGE would be very different in its absence. The spin-orbit-free $D_{\|}^{0}$ and energy bands are shown as dashed gray lines in Fig. 3. The $D_{\|}^{0}$ peak in the conduction band has been suppressed, and a new peak has appeared in the valence band, again associated with a Weyl crossing at $\mathrm{H}$.

In conclusion, the intrinsic CPGE of $p$-Te is strongly affected by the presence of spin-orbit-induced Weyl points at $\mathrm{H}$ and $\mathrm{H}^{\prime}$ near the bottom of the conduction band. The large Berry curvature around those chiral band crossings causes a sign reversal of the photocurrent upon cooling a weakly $p$-doped sample, in agreement with experiment [9]. 

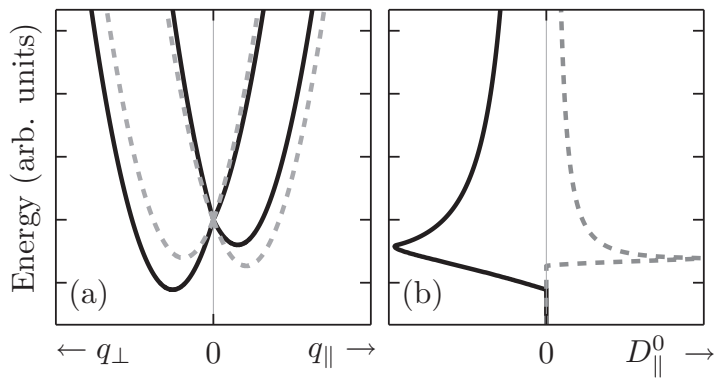

FIG. 4. (a) Energy bands for the anisotropic 3D Rashba model of Eq. (9) with $m_{\|} / m_{\perp}=1$, and $v_{\|} / v_{\perp}=0.6$ (solid line) or $v_{\|} / v_{\perp}=1.1$ (dashed line). The isolated degeneracy is a chiral Weyl point. (b) The quantity $D_{\|}^{0}$ in Eq. (8b) evaluated for the same choices of parameters with $\operatorname{sgn}\left(v_{\|}\right)>0$ (positive chirality). All axes are in arbitrary units.

We emphasize that the Berry-curvature mechanism for the intraband CPGE is different from the one discussed in Ref. [9]. It involves elastic scattering from impurities rather than inelastic phonon scattering, and it relies on the spin-orbit splitting of the conduction subbands that was neglected in that work.

\section{NONLINEAR ANOMALOUS HALL EFFECT}

In tellurium, the nonlinear AHE takes the form of an inplane linear AHE proportional to the current density flowing along the trigonal axis. Taking $j_{\|}=1000 \mathrm{~A} / \mathrm{cm}^{2}$ as a reference value [4,5], the current-induced $\mathrm{AHC}$ is given by [see Eq. (B7)]

$$
\sigma_{x y}^{\mathrm{A}}\left(j_{\|}=1000 \mathrm{~A} / \mathrm{cm}^{2}\right) \approx \frac{0.116 D_{\|}}{C_{\|}(\mathrm{A} / \mathrm{cm})} \mathrm{S} / \mathrm{cm} .
$$

The AHC calculated from Eq. (10) is plotted versus temperature in Fig. 5 at three different doping levels. At high doping it decreases monotonically with temperature, while at low doping it drops to negative values above $220 \mathrm{~K}$ (due to the sign change in $D_{\|}$discussed in the previous section) and then approaches zero from below. Between 50 and $170 \mathrm{~K}$, the AHC is only weakly dependent on $N_{a}$ over a wide doping range. This is due to a near cancellation between the strong dependencies of $D_{\|}$ and $C_{\|}$on $N_{a}$ (see Fig. 6).

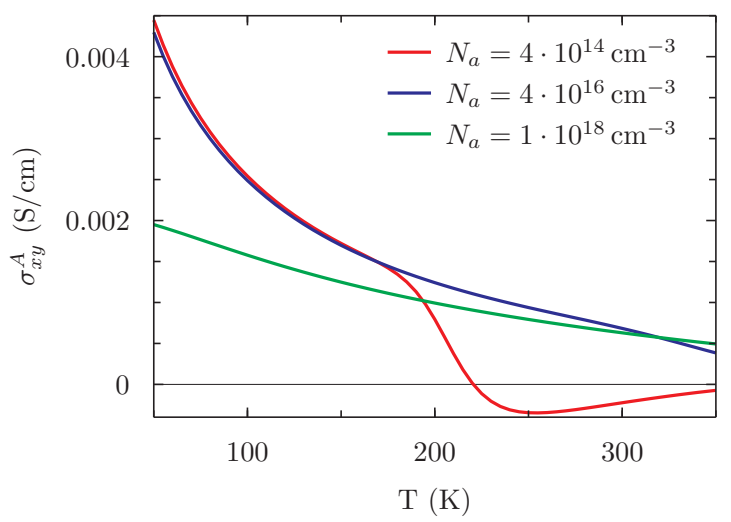

FIG. 5. Anomalous Hall conductivity induced in right-handed Te by a current density $j_{\|}=1000 \mathrm{~A} / \mathrm{cm}^{2}$ [Eq. (10)], plotted versus temperature at different acceptor concentrations.
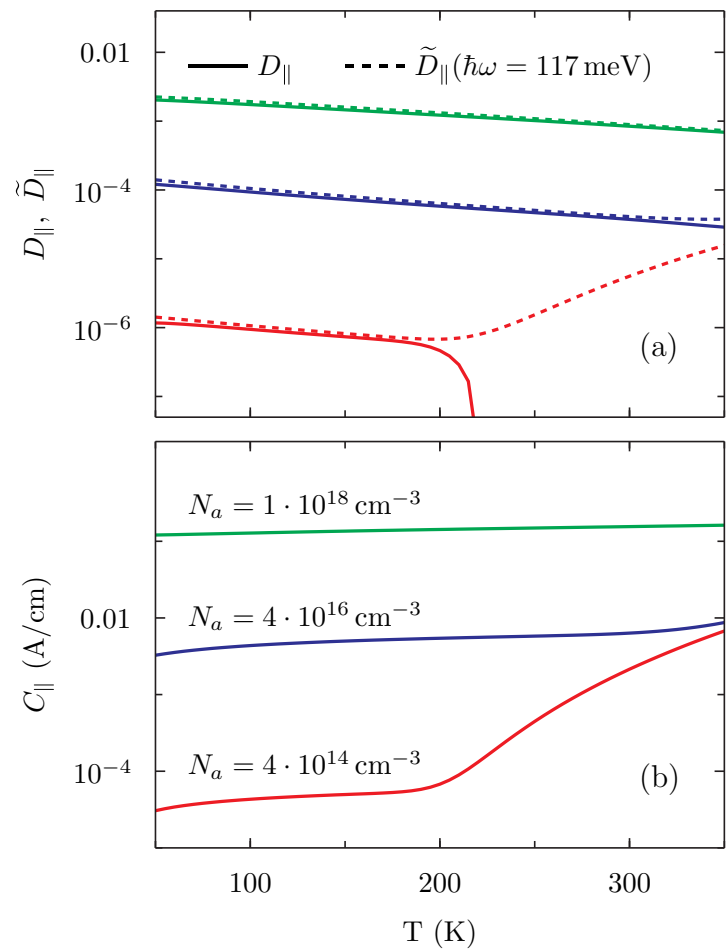

FIG. 6. (a) The quantities $D_{\|}$[Eq. (2)] and $\widetilde{D}_{\|}$[Eq. (12)] in righthanded Te, plotted versus temperature on a semilogarithmic scale for different acceptor concentrations. The strong dip in $\log D_{\|}$around $220 \mathrm{~K}$ at $N_{a}=4 \times 10^{14} \mathrm{~cm}^{-3}$ signals the sign change in $D_{\|}$seen in Fig. 2. (b) The quantity $C_{\|}$[Eq. (B6)].

The current-induced AHC displayed in Fig. 5 does not exceed $5 \times 10^{-3} \mathrm{~S} / \mathrm{cm}$, which is probably too small to be detected (it is five orders of magnitude smaller than the spontaneous AHC of bcc Fe [1]). Nevertheless, the associated Faraday rotation has been observed in the infrared $[4,5]$. The analysis of that effect will occupy us in the next section.

\section{KINETIC FARADAY EFFECT}

So far, $p$-Te is the only material for which the kFE has been measured. The first observation was reported in Ref. [4], and new measurements were taken in Ref. [5]. These works established that the current-induced change in rotatory power $(\Delta \rho)$ is linear in $j_{\|}$up to at least $\pm 1500 \mathrm{~A} / \mathrm{cm}^{2}$, and that $\Delta \rho$ has the opposite (same) sign as the natural rotatory power $\rho_{0}$ when light travels parallel (antiparallel) to the current.

We have calculated $\Delta \rho$ from the following expression, derived in Appendix C3,

$$
\Delta \rho\left(\omega, j_{\|}\right)=\operatorname{sgn}\left(q_{\|}\right) \frac{\mathfrak{a} \widetilde{D}_{\|}(\omega) j_{\|}}{n_{\perp}(\omega) C_{\|}}
$$

(our sign convention for optical rotation is specified in Appendix C 1 ). Here $q_{\|}$is the wave vector of light, and $n_{\perp}$ is the index of refraction; we used the value $n_{\perp}=5.15$ calculated from Eqs. (C7) and (C22), which is slightly higher than the experimental value of 4.8 [30]. When light travels parallel to the current $\left[\operatorname{sgn}\left(q_{\|}\right)=\operatorname{sgn}\left(j_{\|}\right)\right], \Delta \rho$ has the same sign as the 
TABLE I. Natural rotatory power (in units of $\mathrm{rad} / \mathrm{cm}$ ), and current-induced change in rotatory power divided by the current density (in units of $10^{-5} \mathrm{rad} \mathrm{cm} / \mathrm{A}$ ) at $\hbar \omega=0.117 \mathrm{eV}$ and $T=$ $77 \mathrm{~K}$ for two different doping concentrations. The sign of $\Delta \rho / j_{\|}$ corresponds to light propagating in the positive direction along the trigonal axis $\left[\operatorname{sgn}\left(q_{\|}\right)>0\right.$ in Eq. $\left.(11)\right]$.

\begin{tabular}{lccc}
\hline \hline & $\rho_{0}$ & $\Delta \rho / j_{\|}$ & Handedness \\
\hline Expt. & $1.57 \pm 0.03^{\mathrm{a}}$ & $-9.5 \pm 0.4,^{\mathrm{b}}-6^{\mathrm{c}}$ & Unknown \\
Theory & -0.86 & $4.5,4$ & Right-handed
\end{tabular}

${ }^{\mathrm{a}}$ Ref. [31], undoped samples.

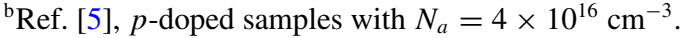

${ }^{\mathrm{c}}$ Ref. [4], $p$-doped samples with $N_{a}=1.5 \times 10^{17} \mathrm{~cm}^{-3}$.

quantity $\widetilde{D}_{\|}(\omega)$ defined by

$$
\widetilde{D}_{a b}(\omega)=\int[d \boldsymbol{k}] \sum_{n} \frac{\partial E_{k n}}{\partial k_{a}} \widetilde{\Omega}_{k n}^{b}(\omega)\left(-\frac{\partial f_{0}}{\partial E}\right)_{E=E_{k n}},
$$

a finite-frequency generalization of Eq. (2) obtained by replacing $\boldsymbol{\Omega}_{\boldsymbol{k} n}$ therein with $\widetilde{\boldsymbol{\Omega}}_{\boldsymbol{k} n}(\omega)$ given by Eq. (C20).

In addition to $\Delta \rho$, we have calculated the rotatory power $\rho_{0}$ caused by the natural optical activity of Te at $j_{\|}=0$. We used the formalism described in Appendix C 2 a to evaluate $\rho_{0}$ ignoring the influence of doping (the effect of doping on $\rho_{0}$ will be analyzed in Sec. VII, where it is shown to be negligible at the doping levels used in the kFE measurements [4,5]).

Table I shows the calculated values of $\rho_{0}$ and $\Delta \rho / j_{\|}$ alongside the experimental ones, measured on samples of unknown handedness. In agreement with experiment, we find that $\Delta \rho$ has the opposite sign from $\rho_{0}$ when light travels parallel to the current (we defer the discussion of absolute signs to Sec. VC). The calculated $\left|\rho_{0}\right|$ and $|\Delta \rho|$ are smaller by roughly a factor of two compared to the measured values, which can be considered a fair level of agreement. The calculated $|\Delta \rho|$ decreases only slightly as $N_{a}$ is increased from $4 \times 10^{16}$ to $1.5 \times 10^{17} \mathrm{~cm}^{-3}$. The larger decrease seen in the experimental values was attributed in Ref. [5] to technical differences relative to Ref. [4].

At $j_{\|}=1000 \mathrm{~A} / \mathrm{cm}^{3}, \Delta \rho$ is about five orders of magnitude smaller than the spontaneous Faraday rotatory power of bcc Fe [32]. This is the same difference in orders of magnitude that was found in the previous section for the AHC. However, the smallness of the kFE is compensated by the high transparency of Te in the infrared, which allows one to measure the optical rotation across a cm-sized sample $[4,5]$, compared to $\sim 10^{-6}$ cm-thick iron films [32].

\section{A. Doping and temperature dependence}

Figure 7 shows a weak doping dependence of $\Delta \rho$ at low doping between 50 and $170 \mathrm{~K}$, in good agreement with the experimental data in Ref. [34] (p. 27), and a monotonic decrease with temperature. The decrease is by a factor of three to four between 77 and $300 \mathrm{~K}$, in agreement with an earlier theoretical estimate [35]. Apart from the previously mentioned overall factor of two which at present we cannot account for, the calculated $\Delta \rho$ agrees rather well with the experimental data reported in Refs. [33] and [34] (p. 35), as indicated by the open circles in Fig. 7.

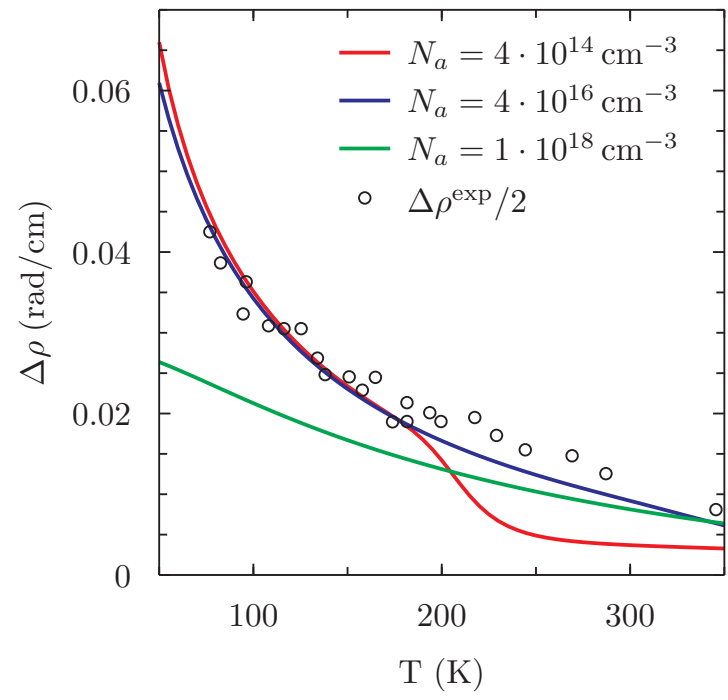

FIG. 7. Temperature dependence of the change in the rotatory power of right-handed Te induced by a current density of $1000 \mathrm{~A} / \mathrm{cm}^{2}$. The optical frequency is $\hbar \omega=0.117 \mathrm{eV}, N_{a}$ is the doping level, and the sign of $\Delta \rho$ corresponds to light propagating parallel to the current along the trigonal axis. The open circles denote experimental data $[33,34]$ taken at $N_{a}=3.2 \times 10^{16} \mathrm{~cm}^{-3}$, which has been rescaled by a factor of $1 / 2$ for comparison purposes.

Even at the lowest doping, $\Delta \rho$ shows no sign change (only a dip) around $220 \mathrm{~K}$. This behavior, which is in contrast to the CPGE and the nonlinear AHE, can be understood from Fig. 6 (a) where at $N_{a}=4 \times 10^{14} \mathrm{~cm}^{-3}$ the quantity $\widetilde{D}_{\|}$ maintains its sign as $T$ goes above $220 \mathrm{~K}$, whereas $D_{\|}$changes sign.

How close $\widetilde{D}_{\|}(\omega)$ is to $D_{\|}$at a given temperature and doping level depends on how close $\widetilde{\Omega}_{k n}(\omega)$ is to $\Omega_{k n}$ in the relevant energy bands, which in turn depends on how $\omega$ compares with $\omega_{m n}$ for the dominant transitions in Eq. (C20). We proceed as in Sec. III, expressing $\widetilde{D}_{\|}(\omega)$ in terms of $\widetilde{D}_{\|}^{0}(\varepsilon, \omega)$ according to Eq. (8). The band-edge behavior of $\widetilde{D}_{\|}^{0}(\varepsilon, \omega)$ and $\widetilde{\Omega}_{\boldsymbol{k} n}^{z}(\omega)$ at $\hbar \omega=0.117 \mathrm{eV}$ is depicted in Fig. 8, to be compared with Fig. 3. In the valence band $\widetilde{\Omega}_{k n}^{z} \approx \Omega_{k n}^{z}$ and $\widetilde{D}_{\|}^{0} \approx D_{\|}^{0}$, because the dominant coupling is with the conduction bands that are separated by more than $0.3 \mathrm{eV}$ (the coupling to the valence band below, which is closer in energy, is suppressed by selection rules [5]). In contrast $\left|\widetilde{\Omega}_{k n}^{z}\right| \ll\left|\Omega_{k n}^{z}\right|$ in the conduction bands, because $0.117 \mathrm{eV}$ is a large energy compared to the Rashba splitting of the coupled subbands. The peak in $\widetilde{D}_{\|}^{0}$ is therefore strongly reduced compared to the peak in $D_{\|}^{0}$, and this is the reason for $\widetilde{D}_{\|}$not changing sign with temperature at low doping in Fig. 6(a).

In conclusion, at the $\mathrm{CO}_{2}$ laser frequency the $\mathrm{kFE}$ is dominated by contributions that to a good approximation can be expressed in terms of the Berry curvature at the top of the valence band. Since this is the same quantity that governs the intrinsic CPGE at low temperatures (Sec. III), one can correlate the sign of $\Delta \rho$ with that of the photocurrent measured on the same sample. When linearly polarized light travels parallel to the current $\left[\operatorname{sgn}\left(q_{\|}\right) j_{\|}>0\right.$ in Eq. (11)], $\Delta \rho$ has the same sign as the photocurrent induced at low temperatures 

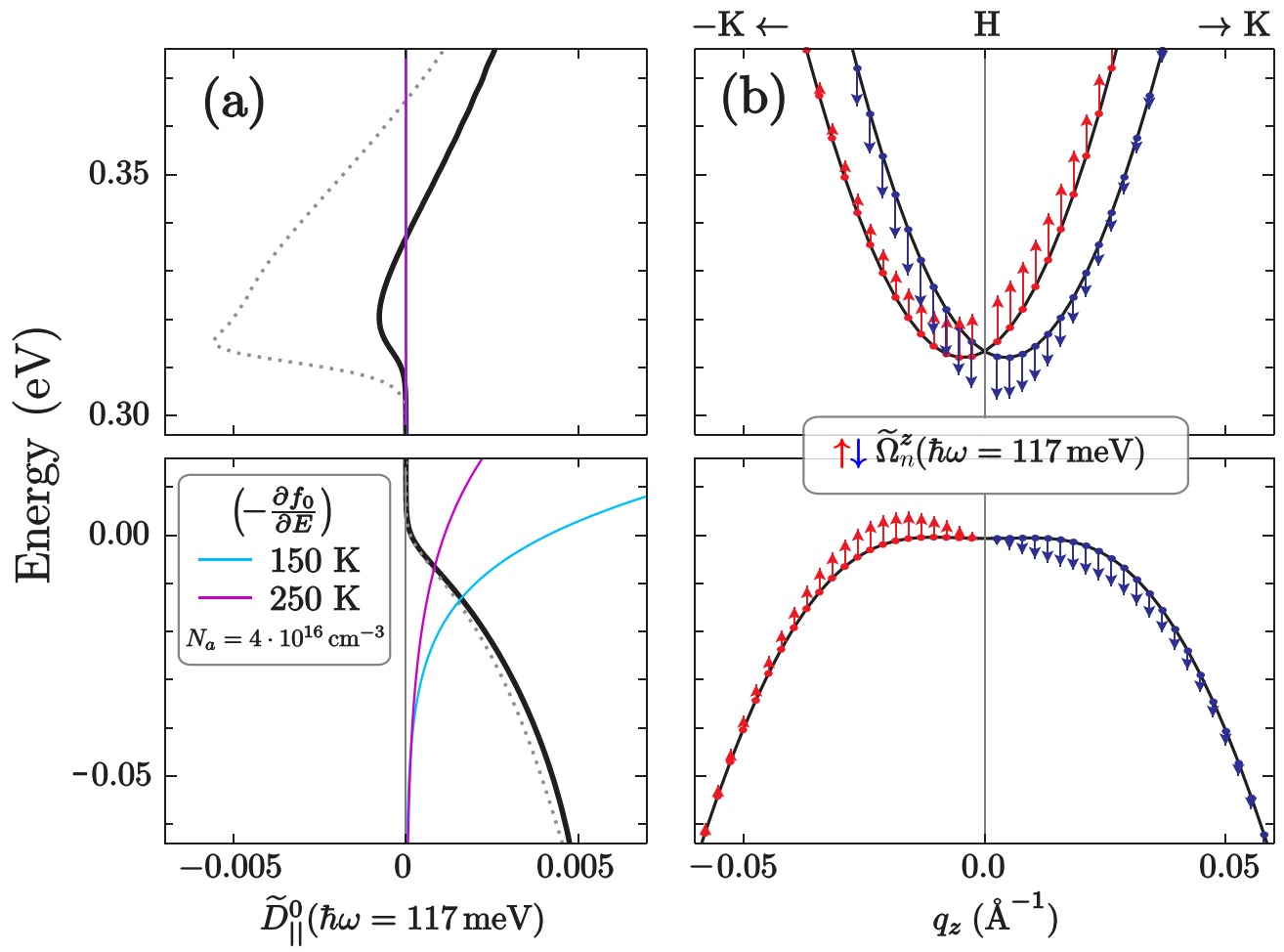

FIG. 8. Microscopic mechanism of the kinetic Faraday effect in right-handed Te at $\hbar \omega=0.117 \mathrm{eV}$. The figure is similar to Fig. 3, but with $D_{\|}^{0}$ replaced by $\widetilde{D}_{a b}^{0}(\omega)$ [the low-temperature limit of Eq. (12)], $\Omega_{k n}^{z}$ by $\widetilde{\Omega}_{k n}^{z}(\omega)$ [Eq. (C20)], and a different doping level when plotting $-\partial f_{0} / \partial E$ in (a). The dotted line in (a) represents $D_{\|}^{0}$, and is identical to the heavy solid line in Fig. 3(a).

by light of positive helicity traveling in the positive direction $\left[\operatorname{sgn}\left(q_{\|}\right) \mathcal{P}_{\text {circ }}>0\right.$ in Eq. (7)].

\section{B. Frequency dependence}

The spectral dependence of the $\mathrm{kFE}$ was investigated in Ref. [5] by taking additional measurements with a $\mathrm{CO}$ laser, which generates radiation of higher frequency than the $\mathrm{CO}_{2}$ laser. These measurements were again taken at $77 \mathrm{~K}$ on samples with $N_{a} \approx 4 \times 10^{16} \mathrm{~cm}^{-3}$.

Between $\hbar \omega=0.117 \mathrm{eV}$ and $\hbar \omega=0.23 \mathrm{eV}, \Delta \rho$ was found to increase by a factor of 1.7. This is significantly less than the increase by a factor of 4.7 in $\rho_{0}$ [31], confirming that current-induced optical rotation and natural optical activity are separate physical effects [5]. Our calculated $\Delta \rho$ and $\rho_{0}$ increased by factors of 1.4 and 5.6, respectively, over the same spectral range, in reasonable agreement with the observed trends.

The calculated $\Delta \rho(\omega)$ is plotted in Fig. 9 at different doping levels and temperatures. At $N_{a}=4 \times 10^{16} \mathrm{~cm}^{-3}$ the spectral dependence is smooth, becoming weaker as the temperature increases. The reason is that at this relatively high doping $\widetilde{D}_{\|}$ is mostly determined by $\widetilde{\Omega}_{k n}$ at the valence-band edge, which depends only weakly on frequency over the subgap spectral range of Fig. 9.

Reducing $N_{a}$ to $4 \times 10^{14} \mathrm{~cm}^{-3}$ has practically no effect on the spectral dependence of $\Delta \rho$ in Fig. 9 at low temperatures, since $\widetilde{D}_{\|}$still originates mostly from the top of the valence band. At $300 \mathrm{~K}$, the contribution from the conduction bands has become significant at this low doping. At frequencies higher than $0.05 \mathrm{eV}$ this leads to a reduction in $\Delta \rho$, due to the opposite signs of $\widetilde{D}_{\|}^{0}$ at the two band edges [Fig. 8(a)]. Below that frequency, the photon energy becomes comparable to the Rasha splitting near the bottom of the conduction band. As a

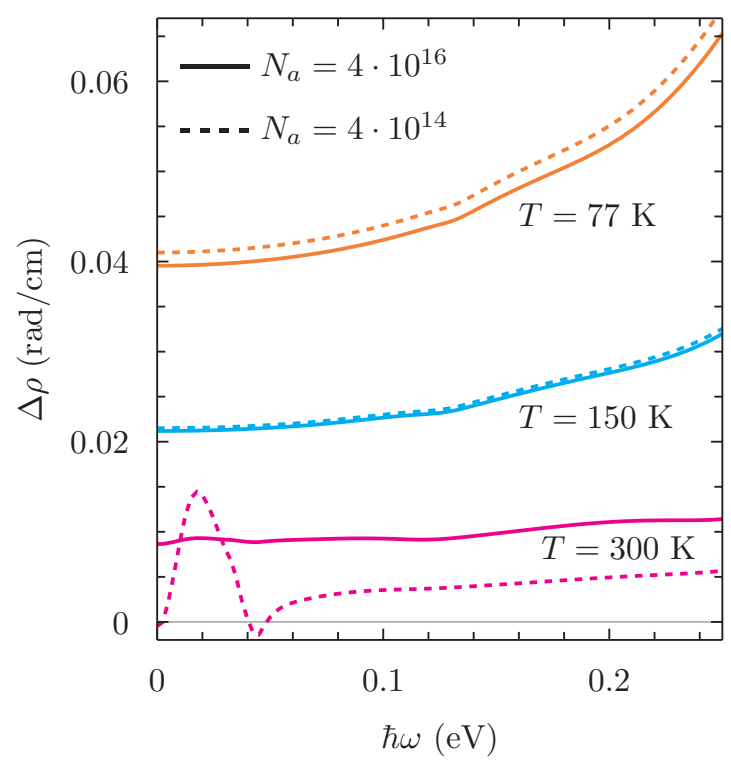

FIG. 9. Frequency dependence of the change in rotatory power induced in right-handed Te by a current density $j_{\|}=1000 \mathrm{~A} / \mathrm{cm}^{2}$, at different temperatures and doping levels. In order to avoid singularities in Eq. $(\mathrm{C} 20)$ at $\omega^{2}=\omega_{k m n}^{2}, \Delta \rho$ is calculated at complex frequencies using $\operatorname{Im}[\hbar \omega]=1 \mathrm{meV}$. 
result, $\Delta \rho$ exhibits a strong dispersion caused by the coupling in Eq. (C20) between the two conduction subbands.

\section{Absolute sign of the optical rotation}

All gyrotropic effects have equal magnitudes and opposite signs for two otherwise identical samples of opposite handedness. Unfortunately the experimental determination of the handedness is particularly difficult for elemental crystals [36], and there are conflicting claims in the literature as to which enantiomorph of trigonal Te rotates the plane of polarization of light in which sense.

We are aware of three studies that tried to establish the handedness of a Te sample, correlating it with the sign of the rotatory power $\rho_{0}$. The first work used etching techniques [37], the second polarized neutron diffraction [38], and the third resonant x-ray diffraction [36]. In Refs. [37,38] it was concluded that the plane of polarization of light rotates in the same sense as the bonded atoms in the spiral chains (with our sign convention, that means $\rho_{0}>0$ for the right-handed structure), and this has become the "accepted wisdom" in the literature $[5,31,39,40]$. However, the authors of the most recent study [36] arrived at the opposite conclusion (see Erratum [41]): Right-handed Te has a negative $\rho_{0}$, in agreement with our calculations.

Let us conclude with a comment on the sign of $\Delta \rho$ calculated in Refs. [4,5] using a $\mathbf{k} \cdot \mathbf{p}$ model for the band-edge states. It was found in those works that $\Delta \rho<0$ when the states with positive (negative) $q_{z}$ at the top of the uppermost valence band are dominated by atomic states with total angular momentum $j_{z}=-3 / 2\left(j_{z}=+3 / 2\right)$. In Ref. [5] that situation was assumed to correspond to right-handed Te, since it leads to $\rho_{0}>0$ [see Eq. (15) in Ref. [28]], in agreement with Refs. [37,38]. However, an examination of our ab initio results leads to the opposite conclusion. For example, the lower panel of Fig. 11(c) shows that in right-handed Te the spin magnetic moment of states near the top of the upper valence band is negative for $q_{z}>0$. In an atomic picture, this corresponds to states with total angular $j_{z}=+3 / 2$ being dominant at positive $q_{z}$. In conclusion, once the $\mathbf{k} \cdot \mathbf{p}$ model is matched to our $a b$ initio wave functions it yields $\rho_{0}<0$ and $\Delta \rho>0$ for right-handed $\mathrm{Te}$, in agreement with our calculations. ${ }^{4}$

\section{KINETIC MAGNETOELECTRIC EFFECT}

Along with the Faraday rotation of transmitted light, the flow of a dc current through a gyrotropic crystal produces a macroscopic magnetization. So far, the intrinsic contribution to this effect has only been calculated for model tight-binding systems $[19,42]$. Our goal in this section is to make quantitative estimates for $p-\mathrm{Te}$, and to provide a microscopic picture for the effect.

\footnotetext{
${ }^{4}$ The $\mathbf{k} \cdot \mathbf{p}$ model of Refs. [4,5] includes spin-orbit coupling in the valence bands only. This is an acceptable approximation, given that the spin-orbit induced Weyl points at the edge of the conduction band do not give a large contribution to the $\mathrm{kFE}$ at the $\mathrm{CO}_{2}$ laser frequency. Recall from Sec. III that this was not the case for the CPGE: Without spin-orbit coupling in the conduction bands, the intrinsic part of the intraband CPGE would not change sign with temperature.
}

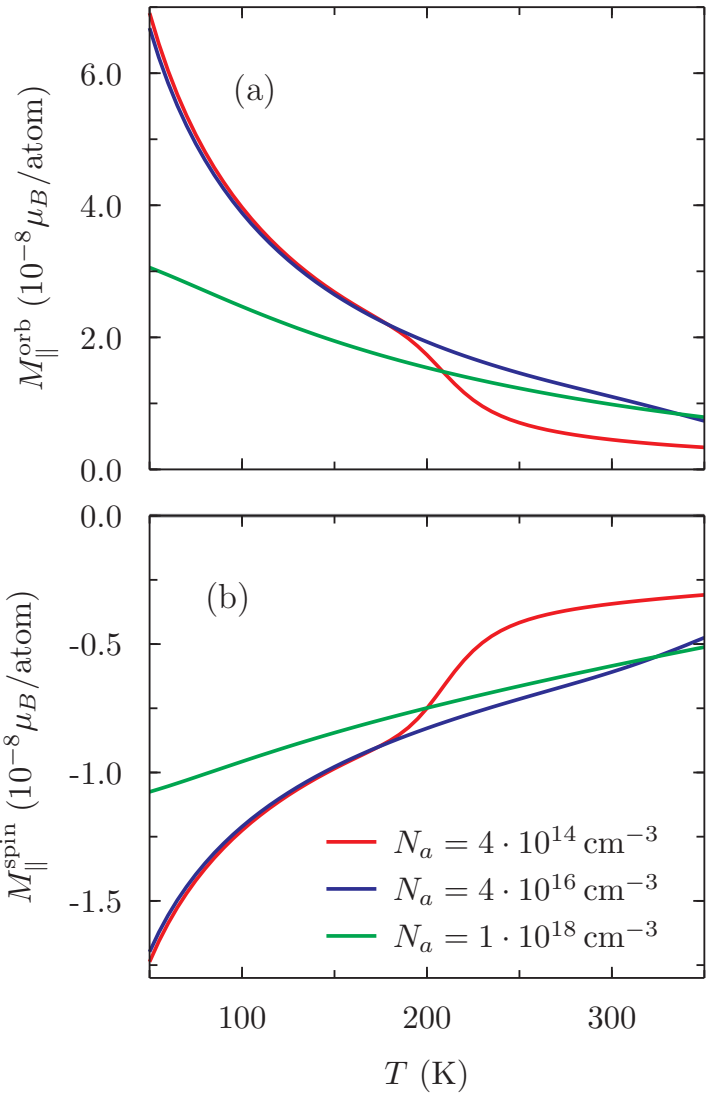

FIG. 10. Temperature dependence of the orbital and spin magnetization induced in right-handed $\mathrm{Te}$, at different acceptor concentrations, by a current density $j_{\|}=1000 \mathrm{~A} / \mathrm{cm}^{2}$.

A current flowing along the trigonal axis induces a parallel magnetization given by (Appendix D)

$$
M_{\|}=-\frac{K_{\|} j_{\|}}{2 \pi C_{\|}} .
$$

The temperature and doping dependence of $M_{\|}$calculated at $j_{\|}=1000 \mathrm{~A} / \mathrm{cm}^{2}$ is shown in Fig. 10. In contrast to $2 \mathrm{D}$ inversion layers where the current-induced magnetization is purely spinlike [16-18], in $p$-Te it has both orbital and spin components, shown separately in Fig. 10. They have opposite signs and comparable magnitudes, with the orbital effect being somewhat larger. ${ }^{5}$ Their magnitudes are $\sim 10^{-8} \mu_{\mathrm{B}}$ /atom, six orders smaller than the spontaneous orbital magnetization in bcc Fe [43] (recall that comparable differences in orders of magnitude relative to bcc Fe were found earlier for the nonlinear AHE and for the $\mathrm{kFE}$ ).

The current-induced spin density at $77 \mathrm{~K}, N_{a}=4 \times$ $10^{16} \mathrm{~cm}^{-3}$, and $j_{\|}=1400 \mathrm{~A} / \mathrm{cm}^{2}$ was estimated in Ref. [5] to be $\sim 560$ spins $/ \mu \mathrm{m}^{3}$. Under the same conditions our calculation yields $561 \mathrm{spins} / \mu \mathrm{m}^{3}$, in a surprisingly perfect agreement. While it may be difficult to directly measure such a small magnetization, indirect evidence for the $\mathrm{kME}$ in $p$-Te

\footnotetext{
${ }^{5}$ Without spin-orbit coupling the bulk kME would be purely orbital $[19,20]$, and we attribute the presence of a comparable spin contribution to the kME to the strong spin-orbit coupling in Te.
} 

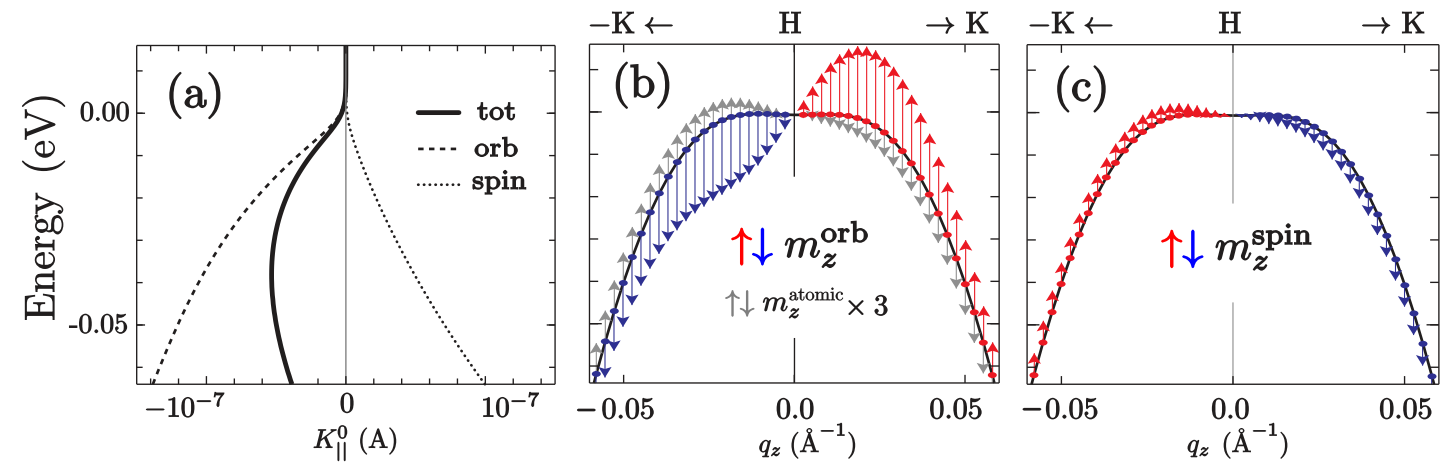

FIG. 11. Microscopic mechanism of the kinetic magnetoelectric effect in right-handed Te. (a) Similar to the lower panel of Fig. 8(a), with $\widetilde{D}_{\|}^{0}$ replaced by $K_{\|}^{0}$ [the low-temperature limit of Eq. (3)]. The total $K_{\|}^{0}$ (heavy solid line) is decomposed into orbital (dashed line) and spin (dotted line) parts. (b) and (c) Like the lower panels of Fig. 8(b), with $\widetilde{\Omega}_{\boldsymbol{k} n}^{z}$ replaced by $m_{\boldsymbol{k} n, z}^{\text {orb }}$ [Eq. (4)] in (b), and by $m_{\boldsymbol{k} n, z}^{\text {spin }}=-\frac{1}{2} g_{s} \mu_{\mathrm{B}}\left\langle\psi_{\boldsymbol{k} n}\left|\sigma_{z}\right| \psi_{\boldsymbol{k} n}\right\rangle$ in (c) - the orbital and spin parts of the intrinsic magnetic moment of a Bloch electron. In (b), the gray arrows denote orbital moments calculated according to Eq. (14).

has already been gathered. In addition to the $\mathrm{kFE}[4,5]$, a current-induced splitting of nuclear magnetic resonance peaks was recently detected [44].

The dominance of the orbital contribution to $M_{\|}$in Fig. 10 implies that it remains positive over the entire temperature range. The signs of $M_{\|}^{\text {orb }}$ and $M_{\|}^{\text {spin }}$ can be understood from Fig. 11. Figure 11(a) shows the quantity $K_{\|}^{0}(\varepsilon)$ [defined in terms of $K_{\|}$in the manner of Eq. (8)] at the top of the valence band, and the signs of its orbital and spin contributions follow from Figs. 11(b) and 11(c), where it can be seen that the $z$ component of the orbital (spin) moments of the band states are antiparallel (parallel) to $\partial E_{k n} / \partial k_{z}$.

The fact that the spin and orbital moments are antiparallel for states in the upper valence band is somewhat surprising. Those states can be approximated as a linear combination of atomic states with total angular momenta $j_{z}= \pm 3 / 2$ [4-6], suggesting parallel spin and orbital moments. This is confirmed by the gray arrows in Fig. 11(b), which show the orbital moments calculated in the atomic-sphere approximation as

$$
m_{\boldsymbol{k} n, z}^{\text {atomic }}=-\mu_{\mathrm{B}} \sum_{i l m} m\left|\left\langle u_{\boldsymbol{k} n} \mid i l m\right\rangle\right|^{2},
$$

where the $|i l m\rangle$ are projectors onto spherical-harmonic states localized on the $i$ th atom in the unit cell, and $\mu_{\mathrm{B}}=e \hbar /\left(2 m_{e}\right)$ is the Bohr magneton. As seen in Fig. 11(b), the moments calculated from Eqs. (4) and (14) differ in both sign and magnitude. This signals a breakdown of the atomic picture of orbital magnetism for states at the top of the valence band, highlighting the need to use the rigorous definition (4) of $\boldsymbol{m}_{\boldsymbol{k} n}^{\text {orb }}$ so as to include itinerant contributions related to the Berry curvature.

In fact, the signs of $m_{\boldsymbol{k} n, z}^{\text {orb }}$ and $\Omega_{\boldsymbol{k} n}^{z}$ are correlated for states in the upper valence band, as can be seen by comparing the spectral decomposition of Eq. (4),

$$
\boldsymbol{m}_{\boldsymbol{k} n}^{\mathrm{orb}}=\frac{e}{2 \hbar} \sum_{m}\left(E_{\boldsymbol{k} m}-E_{\boldsymbol{k} n}\right) \operatorname{Im}\left(\boldsymbol{A}_{\boldsymbol{k} n m} \boldsymbol{A}_{\boldsymbol{k} m n}\right),
$$

with that of Eq. (1b) [given by Eq. (C20) at $\omega=0$ ], and recalling from Sec. V A that the upper valence band couples most strongly to the lower conduction subbands, for which $E_{k m}-E_{k n}>0$. This analysis suggests that $m_{k n, z}^{\text {orb }}$ and $\Omega_{k n}^{z}$ should be antiparallel, which is indeed the case: Compare Fig. 11(b) with Fig. 3(b).

\section{NATURAL OPTICAL ACTIVITY OF DOPED TELLURIUM}

The theoretical value of $\rho_{0}$ in Table I was calculated for undoped Te, and here we analyze how it changes under doping. We consider two effects: the doping dependence of the interband contribution, and the appearance in doped samples of an intraband contribution, whose mechanism is closely related to that of the $\mathrm{kME}[20,21]$. We calculate both effects at $77 \mathrm{~K}$, for the $\mathrm{CO}_{2}$ laser frequency.

We begin with the doping dependence of the interband rotatory power, which can be taken into account by replacing $\sum_{n, l}^{o, e}$ with $\sum_{n, l} f_{k n}\left(1-f_{k l}\right)$ in Eq. (C12) (see Ref. [40]). As shown by the dashed line in Fig. 12, $\rho_{0}^{\text {inter }}$ remain negative over the entire doping range. At first its magnitude decreases slightly with increasing $N_{a}$, due to a depopulation of the upper valence band that blocks some of the interband transitions

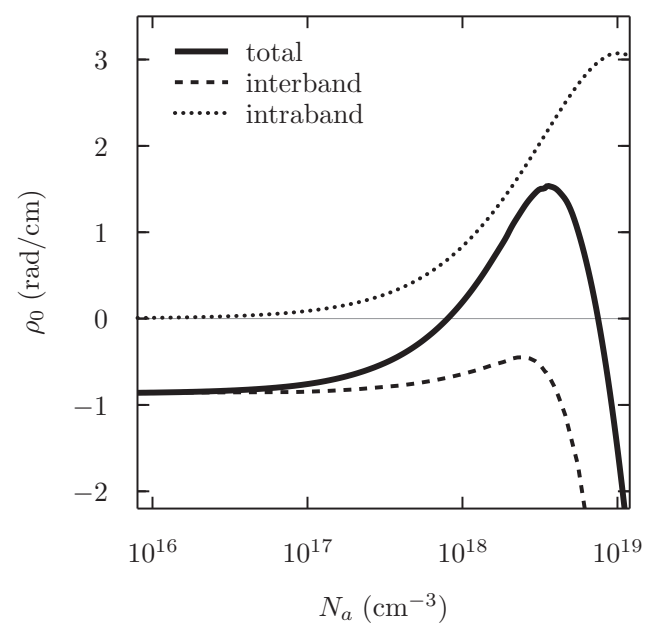

FIG. 12. Doping dependence of the natural rotatory power of right-handed Te at $77 \mathrm{~K}$ and $\hbar \omega=0.117 \mathrm{eV}$, decomposed into interband and intraband contributions. 
[40]. It reaches a minimum at $N_{a} \approx 2.5 \times 10^{18} \mathrm{~cm}^{-3}$, and then increases rapidly in magnitude. The rapid increase is caused by transitions between the two upper valence bands, which become possible at high doping [40]. Although the matrix elements for such transitions are small $[5,40]$, along the HA line the band separation is close to the $\mathrm{CO}_{2}$ laser frequency of $\hbar \omega=$ $0.117 \mathrm{eV}$ [see Fig. 1(b)], producing a resonant enhancement.

We now turn to the intraband rotatory power, shown as the dotted line in Fig. 12. In Appendix C 2 b we obtained, following Refs. [20,21],

$$
\begin{aligned}
\rho_{0}^{\text {intra }}(\omega) & =\frac{\omega^{2} \tau^{2}}{1+\omega^{2} \tau^{2}} \rho_{0}^{\text {clean }}, \\
\rho_{0}^{\text {clean }} & =-\frac{4 \pi \mathfrak{a}}{e c} K_{\perp},
\end{aligned}
$$

with $K_{\perp}$ given by Eqs. (3) and (6). Using the values of $\tau\left(N_{a}, T\right)$ from Ref. [26], we conclude that up to $N_{a}=10^{20} \mathrm{~cm}^{-3}$ the "clean-limit" condition $\omega \tau \gg 1$ is satisfied at the $\mathrm{CO}_{2}$ laser frequency and room temperature (and below). Thus, $\rho_{0}^{\text {intra }} \approx \rho_{0}^{\text {clean }}$ over the entire range of Fig. $12 . \rho_{0}^{\text {intra }}$ has the opposite sign compared to $\rho_{0}^{\text {inter }}$, and a negligible magnitude at low doping. But while $\left|\rho_{0}^{\text {inter }}\right|$ initially decreases as the doping level increases, $\left|\rho_{0}^{\text {intra }}\right|$ increases (more or less linearly) with $N_{a}$. Interestingly, between $8 \times 10^{17}$ and $7.5 \times 10^{18} \mathrm{~cm}^{-3}$ the competition between the two contributions results in a sign reversal of $\rho_{0}$.

\section{SUMMARY}

In summary, we have carried out a combined ab initio study of several gyrotropic effects in $p$-doped Te. ${ }^{6}$ The motivation was provided by recent theoretical developments that recognized the central role played by the Berry curvature and by the intrinsic orbital moment in the description of such effects in the semiclassical regime of low frequencies compared to the band splittings. This prompted us to revisit the pioneering infrared measurements of the CPGE $[8,9]$ and $\mathrm{kFE}[4,5]$ in bulk Te.

We found that the intrinsic mechanism for the intraband CPGE [12-14] accounts for the observed sign reversal of the CPGE with temperature, and that the sign reversal is caused by the presence of Berry-curvature monopoles (Weyl points) at the bottom of the conduction band. This provides an interesting example of the way in which Weyl points can influence physical observables in semiconductors.

Regarding the natural and current-induced optical rotation ( $\mathrm{kFE}$ ), our calculations give rotatory powers whose magnitudes are within a factor of two of the measured ones. In agreement with experiment [5], we find that $\Delta \rho$ and $\rho_{0}$ have opposite signs when light propagates in the same direction as the current.

As for the absolute sign of $\rho_{0}$, we find that in undoped samples the plane of polarization rotates in the opposite sense to the bonded atoms in the spiral chains. This contradicts the

\footnotetext{
${ }^{6}$ The computer code developed for this project was written as a module of the WANNIER 90 package $[45,46]$, and will be made publicly available in a forthcoming release.
}

result of early attempts to determine the handedness of a Te sample [37,38], but agrees with the most recent experimental determination [36,41]. We also predict a sign reversal of $\rho_{0}$ over a significant doping range, due to the competition between interband and intraband contributions to the natural optical activity.

In order to compare our fully quantum-mechanical calculation of $\Delta \rho$ with the semiclassical limit, the result was expressed in terms of a quantity $\widetilde{\boldsymbol{\Omega}}_{k n}(\omega)$ that reduces to the Berry curvature at $\omega=0$. We found that at the $\mathrm{CO}_{2}$ laser frequency, $\widetilde{\boldsymbol{\Omega}}_{\boldsymbol{k} n}(\omega)$ at the top of the valence band is very close to $\boldsymbol{\Omega}_{\boldsymbol{k} n}$. Hence, the low-temperature kFE is well described by the same Berry-curvature parameter $D_{\|}$that governs the intrinsic CPGE. This leads to a definite sign relation between the two effects, which could be tested by measuring both on the same sample.

We have also provided estimates for the magnitudes of other gyrotropic effects that have not yet been observed, such as the nonlinear AHE and the kME. Our estimates indicate that those effects are rather small in $p$-Te. However, a recent study predicted a sizable nonlinear AHE in Weyl semimetals [47].

In closing, we hope that the present work will stimulate further experimental and theoretical work exploring the role of the $k$-space Berry curvature, intrinsic orbital moment, and Weyl points in connection with gyrotropic effects in bulk crystals.

Note added: After this work was submitted, a complementary theoretical study of the kME in $p$-Te appeared [48]. The authors used a $\mathbf{k} \cdot \mathbf{p}$ model to investigate extrinsic as well as intrinsic contributions to the kFE. For doping concentrations up to a few $10^{17} \mathrm{~cm}^{-3}$ they find that the latter are dominant, with the same magnitude and sign as reported here.

\section{ACKNOWLEDGMENTS}

We acknowledge support from Grant No. FIS2016-77188-P from the Spanish Ministerio de Economía y Competitividad, Grant No. CIG-303602 from the European Commission, and from Elkartek Grant No. KK-2016/00025. We would like to thank V. A. Shalygin for useful discussions.

\section{APPENDIXES}

In Appendixes A-D we review the theory of the various gyrotropic effects considered in the main text. The photogalvanic effect is treated in Appendix A, the nonlinear AHE in Appendix $\mathrm{B}$, optical rotation in Appendix C, and the kinetic magnetoelectric effect in Appendix D. Concerning the microscopic theory of these effects, our aim is to present a coherent picture based on a small number of basic ingredients (Berry connections, curvatures, and intrinsic magnetic moments). We only consider the "intrinsic" contributions that can be calculated from the electronic structure of the pristine crystal supplemented by a phenomenological relaxation time $\tau$. We therefore neglect extrinsic effects due to skew-scattering and side-jump processes at impurities [12,49]. Finally, Appendix E describes some technical details of our Wannier-based numerical scheme. 


\section{APPENDIX A: PHOTOGALVANIC EFFECT}

\section{Phenomenology}

Consider an oscillating electric field,

$$
\mathcal{E}(\boldsymbol{r}, t)=\operatorname{Re}\left[\mathcal{E}(\omega) e^{i(\boldsymbol{q} \cdot \boldsymbol{r}-\omega t)}\right] .
$$

The current density induced at second order in the field amplitude can be written as [14]

$$
\begin{aligned}
j_{a}(t) & =\operatorname{Re}\left(j_{a}^{0}+j_{a}^{2 \omega} e^{-i 2 \omega t}\right), \\
j_{a}^{0} & =\frac{1}{2} \sigma_{a b c}(\omega) \mathcal{E}_{b}(\omega) \mathcal{E}_{c}^{*}(\omega), \\
j_{a}^{2 \omega} & =\frac{1}{2} \sigma_{a b c}(\omega) \mathcal{E}_{b}(\omega) \mathcal{E}_{c}(\omega) .
\end{aligned}
$$

Equations (A2b) and (A2c) describe a dc photocurrent and a second-harmonic current, respectively.

Writing $\sigma_{a b c}=\lambda_{a b c}+\gamma_{a b c} \quad$ and $\quad \mathcal{E}_{b} \mathcal{E}_{c}^{*}=\operatorname{Re}\left(\mathcal{E}_{b} \mathcal{E}_{c}^{*}\right)+$ $i \operatorname{Im}\left(\mathcal{E}_{b} \mathcal{E}_{c}^{*}\right)$, where the first and second terms in these expressions are, respectively, symmetric and antisymmetric under $b \leftrightarrow c$, Eq. (A2b) becomes

$$
j_{a}^{0}=\frac{1}{2}\left\{\lambda_{a b c} \operatorname{Re}\left(\mathcal{E}_{b} \mathcal{E}_{c}^{*}\right)-\gamma_{a b} \operatorname{Im}\left(\mathcal{E} \times \mathcal{E}^{*}\right)_{b}\right\},
$$

where $\gamma_{a b}=-i \epsilon_{b c d} \gamma_{a c d} / 2=-i \epsilon_{b c d} \sigma_{a c d} / 2$. The first (second) term describes the linear (circular) photogalvanic effects. $\lambda_{a b c}$ transforms like the piezoelectric tensor, and $\gamma_{a b}$ like the gyration tensor $[10,11]$.

\section{Berry-curvature ("intrinsic") contributions}

The intrinsic intraband contribution to the nonlinear conductivity of a nonmagnetic crystal can be expressed in terms of the tensor $D$ in Eq. (2) as [14]

$$
\sigma_{a b c}=-\frac{e^{3} \tau_{\omega}}{\hbar^{2}} \epsilon_{a d c} D_{b d},
$$

where

$$
\tau_{\omega}=\frac{\tau}{1-i \omega \tau} .
$$

Combining Eqs. (A2)-(A4) one finds

$$
\begin{aligned}
\operatorname{Re}\left(j_{a}^{0}\right) & =j_{a}^{\mathrm{LPGE}}+j_{a}^{\mathrm{CPGE}}, \\
j_{a}^{\mathrm{LPGE}} & =-\frac{e^{3}}{2 \hbar^{2}} \operatorname{Re}\left(\tau_{\omega}\right) \epsilon_{a d c} D_{b d} \operatorname{Re}\left(\mathcal{E}_{b} \mathcal{E}_{c}^{*}\right), \\
j_{a}^{\mathrm{CPGE}} & =-\frac{e^{3}}{4 \hbar^{2}} \operatorname{Im}\left(\tau_{\omega}\right) D_{a b} \operatorname{Im}\left(\mathcal{E} \times \mathcal{E}^{*}\right)_{b},
\end{aligned}
$$

where LPGE stands for "linear photogalvanic effect," and $\operatorname{Tr}(D)=0$ was used to eliminate one term from Eq. (A6c) [12].

Consider the CPGE in trigonal Te with light propagating along the trigonal axis. Writing $\boldsymbol{q}=q_{\|} \hat{\boldsymbol{z}}, \mathcal{E}=|\mathcal{E}| \hat{\boldsymbol{e}}$, and $-\operatorname{Im}\left(\boldsymbol{e} \times \boldsymbol{e}^{*}\right)=\mathcal{P}_{\text {circ }} \hat{\boldsymbol{q}}$ where $\mathcal{P}_{\text {circ }}$ is the degree of circular polarization, and defining the intensity of incident light as $I_{0}=c \epsilon_{0}|\mathcal{E}|^{2} / 2$, Eq. (A6c) becomes

$$
j_{\|}^{\mathrm{CPGE}}=\operatorname{sgn}\left(q_{\|}\right)\left(2 \pi \mathfrak{a} \mathcal{P}_{\text {circ }} D_{\|}\right) \operatorname{Im}\left(\tau_{\omega}\right) \frac{e I_{0}}{\hbar},
$$

where $\mathfrak{a}=e^{2} /\left(4 \pi \epsilon_{0} \hbar c\right)$ is the fine-structure constant. For positive helicity $\left(\mathcal{P}_{\text {circ }}>0\right)$, the current flows parallel (antiparallel) to the light beam when $D_{\|}>0\left(D_{\|}<0\right)$.

\section{APPENDIX B: NONLINEAR ANOMALOUS HALL EFFECT}

In the $\omega \rightarrow 0$ limit the total current from Eqs. (A2) and (A6) becomes

$$
j_{a}=-\frac{e^{3} \tau}{\hbar^{2}} \epsilon_{a d c} D_{b d} \mathcal{E}_{b} \mathcal{E}_{c},
$$

with equal parts coming from the second-harmonic and LPGE currents (the CPGE vanishes at $\omega \rightarrow 0$ ). Since $\mathcal{E} \cdot \boldsymbol{j}=0$, Eq. (B1) describes a nonlinear anomalous Hall current [14].

It is instructive to obtain Eq. (B1) by replacing $f_{0}$ in Eq. (1) with the change in the distribution function at linear order in an applied static field,

$$
\Delta f \equiv f-f_{0}=-e \tau \mathcal{E} \cdot \boldsymbol{v}_{k n}\left(-\frac{\partial f_{0}}{\partial E}\right)_{E=E_{k n}} .
$$

Doing so yields

$$
\Delta \sigma_{a b}^{\mathrm{A}}=\frac{e^{3} \tau}{\hbar^{2}} \epsilon_{a b d} D_{c d} \mathcal{E}_{c}
$$

for the field-induced AHC, in agreement with Eq. (B1).

Inserting Eq. (5) for the tensor $D$ in Te into Eq. (B1) for the current, we obtain

$$
\begin{aligned}
j_{x} & =\frac{3 e^{3} \tau}{2 \hbar^{2}} D_{\|} \mathcal{E}_{z} \mathcal{E}_{y}, \\
j_{y} & =-\frac{3 e^{3} \tau}{2 \hbar^{2}} D_{\|} \mathcal{E}_{z} \mathcal{E}_{x}, \\
j_{z} & =0 .
\end{aligned}
$$

The nonlinear current flows in the plane perpendicular to the trigonal axis, and the effect can be viewed as an in-plane linear AHE induced by the out-of-plane field component $\mathcal{E}_{\|} \equiv \mathcal{E}_{z}$. The effective field-induced AHC is

$$
\sigma_{x y}^{\mathrm{A}}\left(\mathcal{E}_{\|}\right)=\frac{3 e^{3} \tau}{2 \hbar^{2}} D_{\|} \mathcal{E}_{\|}=\frac{3 e^{3}}{2 \hbar^{2}} \frac{D_{\|}}{\sigma_{\|} / \tau} j_{\|},
$$

where in the second equality we inverted Ohm's law to express the result in terms of $j_{\|}$(the nonzero components of the Ohmic conductivity are $\sigma_{\perp} \equiv \sigma_{x x}=\sigma_{y y}$ and $\sigma_{\|} \equiv \sigma_{z z}$ ). In the constant relaxation-time approximation we have $\sigma_{\|} / \tau=(2 \pi e / \hbar) C_{\|}$, with

$$
C_{\|}=\frac{e}{h} \int[d \boldsymbol{k}] \sum_{n}\left(\frac{\partial E_{\boldsymbol{k} n}}{\partial k_{z}}\right)^{2}\left(-\frac{\partial f_{0}}{\partial E}\right)_{E=E_{k n}}
$$

a positive quantity with units of surface current density. With this notation, the current-induced AHC reads

$$
\sigma_{x y}^{\mathrm{A}}\left(j_{\|}\right)=\left(e^{2} / h\right)\left(3 D_{\|} / 2\right)\left(j_{\|} / C_{\|}\right),
$$

where $e^{2} / h$ is the quantum of conductance, $D_{\|}$is dimensionless, and $j_{\|} / C_{\|}$has units of inverse length.

\section{APPENDIX C: OPTICAL ROTATION}

\section{Phenomenology}

The dielectric tensor of trigonal Te has the form [5],

$$
\boldsymbol{\varepsilon}\left(\omega, q_{\|}, j_{\|}\right)=\left(\begin{array}{ccc}
\varepsilon_{\perp} & \varepsilon_{x y}^{\mathrm{A}}\left(\omega, q_{\|}, j_{\|}\right) & 0 \\
-\varepsilon_{x y}^{\mathrm{A}}\left(\omega, q_{\|}, j_{\|}\right) & \varepsilon_{\perp} & 0 \\
0 & 0 & \varepsilon_{\|}
\end{array}\right) .
$$


In equilibrium, the antisymmetric part $\varepsilon_{x y}^{\mathrm{A}}$ responsible for optical rotation is linear in the wave vector $q_{\|}$of light propagating inside the crystal along the trigonal axis. Under a steady current flow, $\varepsilon_{x y}^{\mathrm{A}}$ acquires a new contribution closely related to the nonlinear AHC of Appendix B. It is linear in $j_{\|}$and zeroth order in $q_{\|}$, giving rise to the $\mathrm{kFE}$. (As for the diagonal elements $\varepsilon_{\perp}$ and $\varepsilon_{\|}$, they are independent of $j_{\|}$and $q_{\|}$to linear order.)

Before proceeding further, let us specify our sign convention for optical rotation. We say that the rotatory power $\rho$ is positive when the sense of rotation of the electric field vector is counterclockwise as seen by an observer looking toward the light source. With this choice we have ${ }^{7}$

$$
\rho=\frac{\pi}{\lambda_{0}} \operatorname{Re}\left(n_{-}-n_{+}\right)=\frac{\omega}{2 c} \operatorname{Re}\left(n_{-}-n_{+}\right),
$$

where $\lambda_{0}$ is the wavelength in vacuum, and $n_{+}$and $n_{-}$are the complex indices of refraction for circularly polarized waves of positive and negative helicity, respectively, with polarization vectors given by

$$
\boldsymbol{e}_{ \pm}=\frac{\hat{\boldsymbol{x}} \pm i \operatorname{sgn}\left(q_{\|}\right) \hat{\boldsymbol{y}}}{\sqrt{2}} .
$$

Assuming a sufficiently small current density such that $\left|\varepsilon_{x y}^{A} / \varepsilon_{\perp}\right| \ll 1$, one finds $[5,52]$

$$
n_{-}-n_{+} \approx-\operatorname{sgn}\left(q_{\|}\right) i \frac{\varepsilon_{x y}^{\mathrm{A}} / \varepsilon_{0}}{n_{\perp}},
$$

where $n_{\perp} \equiv \sqrt{\varepsilon_{\perp} / \varepsilon_{0}}$. Converting to conductivities using

$$
\varepsilon_{a b}(\omega)=\varepsilon_{0}\left[\delta_{a b}+\frac{i}{\omega \varepsilon_{0}} \sigma_{a b}(\omega)\right],
$$

we obtain

$$
\rho\left(\omega, j_{\|}\right)=\operatorname{sgn}\left(q_{\|}\right) \frac{\operatorname{Re} \sigma_{x y}^{\mathrm{A}}\left(\omega, j_{\|}\right)}{2 c \varepsilon_{0} n_{\perp}(\omega)}
$$

at nonabsorbing frequencies, with

$$
n_{\perp}(\omega)=\left[1-\frac{1}{\omega \varepsilon_{0}} \operatorname{Im} \sigma_{\perp}(\omega)\right]^{1 / 2} .
$$

In the following, we expand the rotatory power as [5]

$$
\rho\left(\omega, j_{\|}\right)=\rho_{0}(\omega)+\Delta \rho\left(\omega, j_{\|}\right)+\mathcal{O}\left(j_{\|}^{2}\right) .
$$

$\rho_{0}$ is the natural rotatory power at $j_{\|}=0$, and $\Delta \rho\left(j_{\|}\right)$is the change in rotatory power at linear order in $j_{\|}$.

\section{Natural optical rotation}

Natural optical rotation is described by $\sigma_{x y}^{\mathrm{A}}(\boldsymbol{q}, \omega)$ at first order in $q_{z}$, which is conventionally written as [53]

$$
\sigma_{x y}^{\mathrm{A}}(\omega, \boldsymbol{q})=\omega \varepsilon_{0} \gamma_{x y z} q_{z}=\operatorname{sgn}\left(q_{\|}\right) \omega \varepsilon_{0} \gamma_{x y z}\left|q_{\|}\right|,
$$

where $\gamma_{x y z}$ has units of length. Using $\left|q_{\|}\right| / \operatorname{Re} n_{\perp}=\omega / c$, Eq. (C6) becomes [28]

$$
\rho_{0}(\omega)=\frac{\omega^{2}}{2 c^{2}} \operatorname{Re} \gamma_{x y z}(\omega) .
$$

\footnotetext{
${ }^{7}$ Compare with Eq. (2) in Ch. XIV of Ref. [50], where the opposite sign convention for $\rho$ was adopted. Therein, "left-circular polarization" refers to our positive helicity (see also Ref. [51]).
}

Note that the natural rotatory power does not reverse sign with $q_{\|}$. Thus, if a linearly polarized ray travels back and forth inside the material the plane of polarization is unchanged when it returns to the initial point [52,53].

We now turn to the microscopic theory. The natural optical activity of nonconducting crystals is governed by virtual interband transitions $[28,54,55]$, and the rotatory power decreases as $\omega^{2}$ at frequencies well below those of interband transitions. Instead, conducting crystals remain optically active at such low frequencies due to intraband processes [20,21]. Thus, the rotatory power of a conducting crystal is given by

$$
\rho_{0}(\omega)=\rho_{0}^{\text {inter }}(\omega)+\rho_{0}^{\text {intra }}(\omega) .
$$

In the following, both contributions are calculated.

\section{a. Interband natural optical rotation}

Following Ref. [55] we write, with $\partial_{c} \equiv \partial / \partial k_{c}$,

$$
\begin{aligned}
\operatorname{Re} \gamma_{a b c}^{\text {inter }}(\omega)= & \frac{e^{2}}{\varepsilon_{0} \hbar^{2}} \int[d \boldsymbol{k}] \sum_{n, l}^{o, e}, \\
& \times\left[\frac{1}{\omega_{l n}^{2}-\omega^{2}} \operatorname{Re}\left(A_{l n}^{b} B_{n l}^{a c}-A_{l n}^{a} B_{n l}^{b c}\right)\right. \\
& \left.-\frac{3 \omega_{l n}^{2}-\omega^{2}}{\left(\omega_{l n}^{2}-\omega^{2}\right)^{2}} \partial_{c}\left(E_{l}+E_{n}\right) \operatorname{Im}\left(A_{n l}^{a} A_{l n}^{b}\right)\right] .
\end{aligned}
$$

The summations over $n$ and $l$ span the occupied ( $o$ ) and empty (e) states, respectively, $\omega_{l n}=\left(E_{l}-E_{n}\right) / \hbar$, and we omit the $\boldsymbol{k}_{\boldsymbol{k}}$ subscript for brevity. Here

$$
A_{l n}^{a}=i\left\langle u_{l} \mid \partial_{a} u_{n}\right\rangle
$$

is the matrix generalization of the Berry connection appearing in Eq. (1b). Finally, the matrix $B_{n l}^{a c}$ has both orbital and spin contributions given by

$$
B_{n l}^{a c \text { (orb) }}=\left\langle u_{n}\left|\left(\partial_{a} H\right)\right| \partial_{c} u_{l}\right\rangle-\left\langle\partial_{c} u_{n}\left|\left(\partial_{a} H\right)\right| u_{l}\right\rangle,
$$

and

$$
B_{n l}^{a c \text { (spin) }}=-\frac{i \hbar^{2}}{m_{e}} \epsilon_{a b c}\left\langle u_{n}\left|\sigma_{b}\right| u_{l}\right\rangle .
$$

In Te the spin matrix elements contribute less than $0.5 \%$ of the total $\rho_{0}^{\text {inter }}$, and can be safely ignored. Writing $H=$ $\sum_{m}\left|u_{m}\right\rangle E_{m}\left\langle u_{m}\right|$, the orbital matrix elements become

$$
\begin{aligned}
B_{n l}^{a c \text { (orb) }=} & -i \partial_{a}\left(E_{n}+E_{l}\right) A_{n l}^{c} \\
& +\sum_{m}\left\{\left(E_{n}-E_{m}\right) A_{n m}^{a} A_{m l}^{c}-\left(E_{l}-E_{m}\right) A_{n m}^{c} A_{m l}^{a}\right\} .
\end{aligned}
$$

This reduces the calculation of $B^{\text {(orb) }}$ to the evaluation of band gradients and off-diagonal elements of the Berry connection matrix, and both operations can be carried out efficiently in a Wannier-function basis [56].

In our implementation, the summation in Eq. (C16) is restricted to the $s$ and $p$ bands included in the Wannierization procedure (see Appendix E). To check how quickly the calculated $\rho_{0}^{\text {inter }}$ converges with the number of bands, we redid 
the calculation keeping only the four bands (two valence and two conduction) closest to the gap, and found that the value changed by only $10 \%$ compared to a calculation including all $s$ and $p$ states. This is consistent with the conclusion of Ref. [40] that the natural optical activity of Te is contributed mainly by transitions between states near the energy gap.

\section{b. Intraband natural optical rotation}

Here we calculate $\rho_{0}^{\text {intra }}$ following Refs. [20,21]. Combining Eqs. (5a) and (S61) in Ref. [20] and noting that in our notation the tensor $\alpha^{\mathrm{GME}}$ defined therein is given by $-i \omega(e / \hbar) \tau_{\omega} K$, we find

$$
\operatorname{Re} \gamma_{a b c}^{\mathrm{intra}}(\omega)=\frac{e \operatorname{Im} \tau_{\omega}}{\omega \varepsilon_{0} \hbar}\left(\epsilon_{a c d} K_{b d}-\epsilon_{b c d} K_{a d}\right) .
$$

Using Eq. (6) for the tensor $K$ in Te leads to

$$
\operatorname{Re} \gamma_{x y z}^{\text {intra }}(\omega)=-\frac{2 e \operatorname{Im} \tau_{\omega}}{\omega \varepsilon_{0} \hbar} K_{\perp} .
$$

The intraband rotatory power of Eq. (16) is obtained by inserting this expression in Eq. (C10).

\section{Current-induced optical rotation}

Let us now obtain a microscopic expression for $\Delta \rho$ in Eq. (C8), by expanding Eq. (C6) to first order in $j_{\|}$. For that purpose, it is sufficient to expand the tensor $\operatorname{Re} \sigma_{x y}^{\mathrm{A}}(\omega)$ in the numerator. At $j_{\|}=0$ it is given by the following finite-frequency generalization of Eq. (1),

$$
\operatorname{Re} \sigma_{a b}^{\mathrm{A}}(\omega)=-\frac{e^{2}}{\hbar} \int[d \boldsymbol{k}] \sum_{n} f_{0}\left(E_{\boldsymbol{k} n}\right) \epsilon_{a b c} \widetilde{\Omega}_{\boldsymbol{k} n}^{c}(\omega),
$$

where the quantity,

$$
\widetilde{\boldsymbol{\Omega}}_{\boldsymbol{k} n}(\omega)=-\sum_{m} \frac{\omega_{\boldsymbol{k} m n}^{2}}{\omega_{\boldsymbol{k} m n}^{2}-\omega^{2}} \operatorname{Im}\left(\boldsymbol{A}_{\boldsymbol{k} n m} \times \boldsymbol{A}_{\boldsymbol{k} m n}\right),
$$

reduces to the Berry curvature at $\omega=0 .{ }^{8}$

The correction to Eq. (C19) at first order in $j_{\|}$can be obtained by replacing $f_{0}$ therein with $\Delta f$ given by Eq. (B2). Following Appendix B we obtain

$$
\operatorname{Re} \sigma_{x y}^{\mathrm{A}}\left(\omega, j_{\|}\right)=\left(e^{2} / h\right) \widetilde{D}_{\|}(\omega)\left(j_{\|} / C_{\|}\right),
$$

with $\widetilde{D}_{\|}(\omega)$ given by Eq. (12), and inserting this expression in Eq. (C6) we arrive at Eq. (11) for $\Delta \rho$. Note that $\Delta \rho$ reverses sign with $q_{\|}$, contrary to $\rho_{0}$ : Like the conventional Faraday effect [52,53], the kFE is nonreciprocal.

The final step is to determine the refraction index $n_{\perp}$ appearing in Eq. (11). For that purpose, we evaluate the quantity $\operatorname{Im} \sigma_{\perp}(\omega)$ in Eq. (C7) using

$$
\begin{aligned}
\operatorname{Im} \sigma_{\perp}(\omega)= & -\frac{e^{2}}{\hbar} \int[d \boldsymbol{k}] \sum_{n m}^{\prime} f_{0}\left(E_{\boldsymbol{k} n}\right)\left[1-f_{0}\left(E_{\boldsymbol{k} m}\right)\right] \\
& \times \frac{\omega_{\boldsymbol{k} m n}^{2}}{\omega_{\boldsymbol{k} m n}^{2}-\omega^{2}}\left(\left|A_{\boldsymbol{k} n m}^{x}\right|^{2}+\left|A_{\boldsymbol{k} n m}^{y}\right|^{2}\right),
\end{aligned}
$$

\footnotetext{
${ }^{8}$ Contrary to the Berry curvature, the divergence of $\tilde{\boldsymbol{\Omega}}_{k n}(\omega)$ is generally nonzero. As a result, $\tilde{D}(\omega)$ given by Eq. (12) can have a nonzero trace at finite frequencies, i.e., $\tilde{D}_{\|} \neq-2 \tilde{D}_{\perp}$ in Te.
}

where the prime on the summation indicates that the term $m=n$ is excluded. This expression gives the interband contribution to $\operatorname{Im} \sigma_{\perp}(\omega)$. Since at the $\mathrm{CO}_{2}$ laser frequency we have $\omega \tau \gg 1$ across the entire range of temperatures and doping levels considered in our calculations, the intraband (Drude) contribution is negligible.

\section{APPENDIX D: KINETIC MAGNETOELECTRIC EFFECT}

The kME effect in a conducting gyrotropic crystal is described phenomenologically by [20]

$$
\begin{aligned}
& j_{a}^{\boldsymbol{B}}(\omega)=i \omega \alpha_{a b}(\omega) B_{b}(\omega), \\
& M_{a}(\omega)=\alpha_{b a}(\omega) \mathcal{E}_{b}(\omega) .
\end{aligned}
$$

In the limit $\omega \tau \ll 1$ where $\alpha_{a b}(\omega)$ becomes real we have

$$
\begin{aligned}
j_{a}^{\boldsymbol{B}}(t) & =-\alpha_{a b}(0) \dot{B}_{b}(t), \\
M_{a}(t) & =\alpha_{b a}(0) \mathcal{E}_{b}(t),
\end{aligned}
$$

which for an isotropic gyrotropic medium $\left(\alpha_{a b}=\alpha \delta_{a b}\right)$ reduces to Eqs. (1) and (3) of Ref. [15].

It is convenient to introduce a reduced (dimensionless) magnetoelectric tensor,

$$
\alpha_{a b}^{\mathrm{r}}(\omega)=c \mu_{0} \alpha_{a b}(\omega),
$$

in direct analogy with the standard description of magnetoelectric couplings in insulators [57]. The intrinsic part is given in terms of Eqs. (2) and (A5) by

$$
\alpha_{a b}^{\mathrm{r}}(\omega)=-4 \pi \mathfrak{a} \frac{\tau_{\omega}}{e} K_{a b} .
$$

It can be verified that at $\omega=0$ this expression agrees with that obtained in Ref. [19] for the magnetization induced by a static $\mathcal{E}$ field. Specializing to Te and following Appendix B to recast the result in terms of $j_{\|}$, we obtain

$$
M_{\|}=\left(e / 8 \pi^{2} \mathfrak{a}\right)\left(\alpha_{\|}^{\mathrm{r}}(0) / \tau\right)\left(j_{\|} / C_{\|}\right),
$$

which combined with Eq. (D4) becomes Eq. (13).

\section{APPENDIX E: WANNIER INTERPOLATION}

In order to interpolate in $k$ space the energy bands and other quantities (see below), we use the formalism of maximally localized Wannier functions [58,59], as implemented in the WANNIER 90 code package $[45,46]$. We construct four disentangled Wannier functions per tellurium atom and per spin channel, for a total of 24 Wannier functions per cell. The $5 \mathrm{~s}$ and $5 p$ bands of trigonal Te are well separated from the lower $d$ states, and they cross with higher-lying sates only in a small region of the Brillouin zone. Thus we set the outer energy window for the disentanglement procedure [59] from -20 to $+5 \mathrm{eV}$ relative to the valence-band maximum, so as to cover all $s$ and $p$ bands. The inner frozen window spans the range from -20 to $+2.5 \mathrm{eV}$, and we choose atom-centered $s p^{3}$-type trial orbitals for the initial projections. This choice of Wannier functions differs from that of Ref. [24,27], where only $5 p$ states were included in the Wannierization.

The Wannier basis is also used to evaluate the $k$-space quantities entering the expressions for the response tensors, namely: the band gradient $\nabla_{k} E_{k n}$, the Berry curvature $\boldsymbol{\Omega}_{\boldsymbol{k}}$ 
[Eq. (1b)], the intrinsic orbital moment [Eq. (4)], and the off-diagonal elements of the Berry connection matrix $\boldsymbol{A}_{\boldsymbol{k n m}}$ [Eq. (C13)]. The Wannier interpolation of these quantities is described in Refs. $[43,56,60]$.

When evaluating the response tensors, the integrations over the Brillouin zone are performed using a uniform grid of $200 \times 200 \times 200 k$ points. In the case of responses that can be expressed in the form of Eq. (8), when $\varepsilon$ is close to the band gap (no further than $100 \mathrm{meV}$ from the band edges), only $k$ points in the vicinity of $\mathrm{H}$ and $\mathrm{H}^{\prime}$ contribute, due to the factor $\left(-\partial f_{0} / \partial E\right)$ in that equation. In such cases, we use a grid of $200 \times 200 \times 200 k$ points within a small box centered at $H$ that amounts to less than $0.2 \%$ of the entire Brillouin zone, and then multiply the result by two in order to account for $\mathrm{H}^{\prime}$. This allows us to increase the numerical accuracy for $\varepsilon$ near the band gap, which is the energy range that contributes most the response.
[1] N. Nagaosa, J. Sinova, S. Onoda, A. H. MacDonald, and N. P. Ong, Anomalous Hall effect, Rev. Mod. Phys. 82, 1539 (2010).

[2] D. Xiao, M.-C. Chang, and Q. Niu, Berry phase effects on electronic properties, Rev. Mod. Phys. 82, 1959 (2010).

[3] N. B. Baranova, Yu. V. Boddanov, and B. Ya. Zel'Dovich, Electrical analog of the Faraday effect and other new optical effects in liquids, Opt. Commun. 22, 243 (1977).

[4] E. L. Vorob'ev, E. L. Ivchenko, G. E. Pikus, I. I. Farbshtein, V. A. Shalygin, and A. V. Shturbin, Optical activity in tellurium induced by a current, JETP Lett. 29, 441 (1979).

[5] V. A. Shalygin, A. N. Sofronov, E. L. Vorob'ev, and I. I. Farbshtein, Current-Induced spin polarization of holes in tellurium, Phys. Solid State 54, 2362 (2012).

[6] E. L. Ivchenko and G. E. Pikus, New photogalvanic effect in gyrotropic crystals, JETP Lett. 27, 604 (1978).

[7] V. I. Belinicher and B. I. Sturman, The photogalvanic effect in media lacking a center of inversion, Sov. Phys. Usp. 23, 199 (1980).

[8] V. M. Asnin, A. A. Bakun, A. M. Danishevskii, E. L. Ivchenko, G. E. Pikus, and A. A. Rogachev, Observation of a photo-emf that depends on the sign of the circular polarization of the light, JETP Lett. 28, 74 (1978).

[9] V. M. Asnin, A. A. Bakun, A. M. Danishevskii, G. E. Pikus, and A. A. Rogachev, "Circular" photogalvanic effect in optically active crystals, Solid State Commun. 30, 565 (1979).

[10] B. I. Sturman and V. M. Fridkin, The Photovoltaic and Photorefractive Effects in Noncentrosymmetric Materials (Gordon and Breach, Philadelphia, 1992).

[11] E. L. Ivchenko and G. E. Pikus, Superlattices and Other Heterostructures (Springer, Berlin, 1997).

[12] E. Deyo, L. E. Golub, E. L. Ivchenko, and B. Spivak, Semiclassical theory of the photogalvanic effect in non-centrosymmetric systems, arXiv:0904.1917.

[13] J. E. Moore and J. Orenstein, Confinement-Induced Berry Phase and Helicity-Dependent Photocurrents, Phys. Rev. Lett. 105, 026805 (2010).

[14] I. Sodemann and L. Fu, Quantum Nonlinear Hall Effect Induced by Berry Curvature Dipole in Time-Reversal Invariant Materials, Phys. Rev. Lett. 115, 216806 (2015).

[15] L. S. Levitov, Yu. V. Nazarov, and G. M. Eliashberg, Magnetoelectric effects in conductors with mirror isomer symmetry, Sov. Phys. JETP 61, 133 (1985).

[16] V. M. Edelstein, Spin polarization of conduction electrons induced by electric current in two-dimensional asymmetric electron systems, Solid State Commun. 73, 233 (1990).

[17] A. G. Aronov and Y. Lyanda-Geller, Nuclear electric resonance and orientation of carrier spins by an electric field, JETP Lett. 50, 431 (1989).
[18] S. D. Ganichev, M. Trushin, and J. Schliemann, Spin Polarization by Current, in Handbook of Spin Transport and Magnetism, edited by E. Y. Tsymbal and I. Zutic (CRC Press, New York, 2012), p. 487.

[19] T. Yoda, T. Yokoyama, and S. Murakami, Current-induced orbital and spin magnetizations in crystals with helical structure, Sci. Rep. 5, 12024 (2015).

[20] S. Zhong, J. E. Moore, and I. Souza, Gyrotropic Magnetic Effect and the Magnetic Moment on the Fermi Surface, Phys. Rev. Lett. 116, 077201 (2016)

[21] J. Ma and D. A. Pesin, Chiral magnetic effect and natural optical activity in metals with or without Weyl points, Phys. Rev. B 92 235205 (2015).

[22] R. H. Asendorf, Space group of tellurium and selenium, J. Chem. Phys. 27, 11 (1957).

[23] J. Paier, M. Marsman, K. Hummer, G. Kresse, I. C. Gerber, and J. G. Angyán, Screened hybrid density functionals applied to solids, J. Chem. Phys. 124, 154709 (2006).

[24] M. Hirayama, R. Okugawa, S. Ishibashi, S. Murakami, and T. Miyake, Weyl Node and Spin Texture in Trigonal Tellurium and Selenium, Phys. Rev. Lett. 114, 206401 (2015).

[25] V. B. Anzin, M. I. Eremets, Y. V. Kosichkin, A. I. Nadezhdinskii, and A. M. Shirokov, Measurement of the energy gap in tellurium under pressure, Phys. Status Solidi A 42, 385 (1977).

[26] H. Peng, N. Kioussis, and G. J. Snyder, Elemental tellurium as a chiral $p$-type thermoelectric material, Phys. Rev. B 89, 195206 (2014).

[27] S. S. Tsirkin, I. Souza, and D. Vanderbilt, Composite Weyl nodes stabilized by screw symmetry with and without time-reversal invariance, Phys. Rev. B 96, 045102 (2017).

[28] E. L. Ivchenko and G.E. Pikus, Natural optical activity of semiconductors (tellurium). Sov. Phys. Solid State 16, 1261 (1975).

[29] J. K. Asbóth, L. Oroszlányu, and A. Pályi, A Short Course on Topological Insulators (Springer, Berlin, 2016).

[30] G. H. Sherman et al., in Proceedings of the Symposium on Submillimeter Waves (Polytechnic Press, Brooklyn, 1970).

[31] S. Fukuda, T. Shiosaki, and A. Kawabata, Infrared optical activity in tellurium, Phys. Stat. Sol. B 68, K107 (1975).

[32] R. L. Coren and M. H. Francombe, Optical Faraday effect in ferromagnetic and ferrite films, J. Phys. 25, 233 (1964).

[33] A. V. Shturbin and V. A. Shalygin, Temperature dependence of current-induced optical activity of tellurium, Sov. Phys. Semicond. 15, 1309 (1981).

[34] V. A. Shalygin, Optical and photogalvanic effects in bulk semiconductors and two-dimensional structures, St. Petersburg Polytechnic University, 2013 [dissertation in Russian], http://elib.spbstu.ru/dl/2/4754.pdf. 
[35] N. S. Averkiev, On the problem of the temperature-dependence of a current-induced optical activity in tellurium, Sov. Phys. Semicond. 18, 450 (1984).

[36] Y. Tanaka, S. P. Collins, S. W. Lovesey, M. Matsumami, T. Moriwaki, and S. Shin, Determination of the absolute chirality of tellurium using resonant diffraction with circularly polarized X-rays, J. Phys. Condens. Matter 22, 122201 (2010).

[37] A. Koma, E. Takimoto, and S. Tanaka, Etch pits and crystal structure of tellurium, Phys. Status Solidi 40, 239 (1970).

[38] P. J. Brown and J. B. Forsyth, The crystal structure and optical activity of tellurium, Acta Cryst. A52, 408 (1996).

[39] S. Ades and C. H. Champness, Optical activity of tellurium to $20 \mu \mathrm{m}$, J. Opt. Soc. Am. 65, 217 (1975).

[40] H. Stolze, M. Lutz, and P. Grosse, The optical activity of tellurium, Phys. Status Solidi B 82, 457 (1977).

[41] Y. Tanaka, S. P. Collins, S. W. Lovesey, M. Matsunami, T. Moriwaki, and S. Shin, Corrigendum: Determination of the absolute chirality of tellurium using resonant diffraction with circularly polarized X-rays, J. Phys. Condens. Matter 24, 159501 (2012).

[42] T. Yoda, T. Yokoyama, and S. Murakami, Orbital Edelstein effect as a condensed-matter analog of solenoid, arXiv:1706.7702.

[43] M. G. Lopez, D. Vanderbilt, T. Thonhauser, and I. Souza, Wannier-based calculation of the orbital magnetization in crystals, Phys. Rev. B 85, 014435 (2012).

[44] T. Furukawa, Y. Shimokawa, K. Kobayashi, and I. Tetsuaki, Observation of current-induced bulk magnetization in elemental tellurium, Nat. Commun. 8, 954 (2017).

[45] A. A. Mostofi, J. R. Yates, Y.-S. Lee, I. Souza, D. Vanderbilt, and N. Marzari, WANNIER90: A tool for obtaining maximallylocalised Wannier functions, Comput. Phys. Commun. 178, 685 (2008).

[46] A. A. Mostofi, J. R. Yates, G. Pizzi, Y.-S. Lee, I. Souza, D. Vanderbilt, and N. Marzari, An updated version of WANNIER90: A tool for obtaining maximally-localised Wannier functions, Comput. Phys. Commun. 185, 2309 (2014).
[47] Y. Zhang, Y. Sun, and B. Yan, The Berry curvature dipole in Weyl semimetal materials: An ab initio study, Phys. Rev. B 97, 041101(R) (2018).

[48] C. Şahin, J. Rou, J. Ma, and D. A. Pesin, Pancharatnam-Berry phase and kinetic magnetoelectric effect in a three-dimensional helical crystal (Te), arXiv:1712.6586.

[49] J. Rou, C. Şahin, J. Ma, and D. A. Pesin, Kinetic orbital moments and nonlocal transport in disordered metals with nontrivial band geometry, Phys. Rev. B 96, 035120 (2017).

[50] J. F. Nye, Physical Properties of Crystals (Clarendon Press, Oxford, 1985).

[51] J. D. Jackson, Classical Electrodynamics (Wiley, New York, 1999).

[52] J. M. Liu, Principles of Photonics (Cambridge University Press, New York, 2016).

[53] L. D. Landau and E. M. Lifshitz, Electrodynamics of Continuous Media, 2nd ed. (Pergamon Press, Oxford, 1984).

[54] K. Natori, Band theory of the optical activity of crystals, J. Phys. Soc. Jpn. 39, 1013 (1975).

[55] A. Malashevich and I. Souza, Band theory of spatial dispersion in magnetoelectrics, Phys. Rev. B 82, 245118 (2010).

[56] J. R. Yates, X. Wang, D. Vanderbilt, and I. Souza, Spectral and Fermi surface properties from Wannier interpolation, Phys. Rev. B 75, 195121 (2007).

[57] S. Coh, D. Vanderbilt, A. Malashevich, and I. Souza, ChernSimons orbital magnetoelectric coupling in generic insulators, Phys. Rev. B 83, 085108 (2011).

[58] N. Marzari and D. Vanderbilt, Maximally localized generalized Wannier functions for composite energy bands, Phys. Rev. B 56, 12847 (1997).

[59] I. Souza, N. Marzari, and D. Vanderbilt, Maximally localized Wannier functions for entangled energy bands, Phys. Rev. B 65, 035109 (2001).

[60] X. Wang, J. R. Yates, I. Souza, and D. Vanderbilt, Ab initio calculation of the anomalous Hall conductivity by Wannier interpolation, Phys. Rev. B 74, 195118 (2006). 